J. DIFFERENTIAL GEOMETRY

49 (1998) 437-467

\title{
MEASURED FOLIATIONS AND HARMONIC MAPS OF SURFACES
}

\author{
MICHAEL WOLF
}

\section{Introduction}

Fix a Riemannian surface of negative curvature $\left(N^{2}, h\right)$ and a differentiable surface $M_{g}^{2}$ of the same genus $g$ that will host various structures. Also fix a diffeomorphism $f_{0}: M \rightarrow N$. It is well known ([4], [1], [21], [18]) that to every complex structure $\sigma$ on $M$, there is a unique harmonic diffeomorphism $f(\sigma): M(\sigma) \rightarrow(N, h)$ homotopic to $f_{0}: M \rightarrow N$; one is led to consider what other, possibly ostensibly weaker, structures on $M$ might also determine harmonic maps from $M$ to $N$ homotopic to $f_{0}$.

The goal of this paper is to show (Theorem 3.1) that a harmonic map $f\left(\sigma^{\prime}\right):\left(M, \sigma^{\prime}\right) \rightarrow(N, h)$ may be uniquely specified by the initial choice of a class of measured foliations (representing the maximal stretch measured foliation for the harmonic map $f\left(\sigma^{\prime}\right)$ ) rather than an initial choice of complex structure: we observe that a measured foliation may be considered to be a differential-topological object in contrast to the analytical object that a complex structure $\sigma$ represents.

Our proof has aspects of independent interest. In particular, in the proof of uniqueness (§4), from a harmonic map $f:\left(M^{2}, \sigma\right) \rightarrow(N, h)$ of a surface, we construct a naturally associated equivariant (area) minimal map $F:\left(\widetilde{M}^{2}, \tilde{\sigma}\right) \rightarrow(\tilde{N}, \tilde{h}) \times(T, 2 d)$ of the universal cover into the product of the universal cover $(\widetilde{N}, \tilde{h})$ with a real tree $(T, 2 d)$. We show (Theorem 4.3) that for two dimensional negatively curved targets

Received January 6, 1994, and, in revised form, September 25, 1997. Author was partially supported by NSF grants DMS 9300001, 9205540 (SCRMS), and DMS 9022140 (MSRI); Alfred P. Sloan Research Fellow. 
$\left(N^{2}, h\right)$, that this minimal map is stable; we also develop some of the necessary background of this construction and result.

There are a number of contexts for our result. We begin by recalling the Hodge-like theorem of Hubbard and Masur ([12]; see [29] for a Hodge-like proof) which states that on a given Riemann surface $\mathcal{R}$, to each measured foliation $(\mathcal{F}, \mu)$ there exists a unique holomorphic quadratic differential $\Phi_{(\mathcal{F}, \mu)}$ whose horizontal foliation is equivalent to $(\mathcal{F}, \mu)$; again, of course, the measured foliation is topologically defined, while the holomorphic quadratic differential is analytically defined. In our case, the maximal stretch measured foliation is the horizontal stretch foliation of the holomorphic quadratic differential $\Phi(\sigma)=\left(f(\sigma)^{*} h\right)^{(2,0)}$ on $(M, \sigma)$ (all terms to be defined in $\S 2$ ) so that $f(\sigma)_{*} \Phi(\sigma)$ is a quadratic differential on $(N, h)$ satisfying some non-linear equation instead of the Cauchy-Riemann equations. In fact, Tabak [23] has shown that these quadratic differentials are precisely the solution space of a certain system of equations, so that $\left\{f(\sigma)_{*} \Phi(\sigma) \mid \sigma \in T_{g}\right.$, the Teichmüller space of genus $g$ \} are precisely the subsonic, $\rho$-holomorphic quadratic differentials on $(N, h)$ : thus, we show here a Hubbard-Masur theorem for this class of differentials.

The Hubbard-Masur theorem can be thought of as a statement about sections of the cotangent bundle $T^{*} T_{g}$ of Teichmüller space, since $T^{*} T_{g}=Q$, the bundle of holomorphic quadratic differentials on Riemann surfaces. More precisely, the Hubbard-Masur theorem says that each measured foliation $(\mathcal{F}, \mu)$ defines a section $\sigma_{(\mathcal{F}, \mu)}: T_{g} \rightarrow Q$ of $Q$ over $T_{g}$, and that the family of sections $\left\{\sigma_{(\mathcal{F}, \mu)}:[(\mathcal{F}, \mu)] \in M F(M)\right\}$ foliate $Q$. Here we show that the section $s_{h}: T_{g} \rightarrow Q$ of $Q$ defined by $s_{h}([\sigma])=\Phi(\sigma)$ meets each section $\sigma_{(\mathcal{F}, \mu)}$ in a single point; moreover, it then follows from [26, Theorem 3.1] that the family of sections $\left\{s_{h} \mid h\right.$ a hyperbolic metric on $N$ representing a unique point in $\left.T_{g}\right\}$ also foliate $Q$, with each leaf meeting each leaf of the Hubbard-Masur foliation in a single point.

We prove Theorem 3.1 by showing that the map $\mu: T_{g} \rightarrow M F(M)$, defined by sending $[\sigma] \in T_{g}$ to the horizontal measured foliation of $\Phi(\sigma)$, is a homeomorphism. This we prove in steps, by showing that the map $\mu$ is (i) continuous, (ii) surjective, and (iii) injective. As noted above, some features of the proof are of independent interest, so we review them now. The first step, continuity, is straightforward. To show surjectivity, we consider the function $E(\sigma)+2 L_{\mu}(\sigma): T_{g} \rightarrow \mathbf{R}$ on Teichmüller space, where $E(\sigma)$ refers to the energy of the map $f(\sigma):(M, \sigma) \rightarrow(N, h)$, and $L_{\mu}(\sigma)$ refers to the extremal length of the measured foliation $\mu$ on the 
Riemann surface $(M, \sigma)$. We find a complex structure $\sigma$ for which the maximal stretch foliation of $w(\sigma)$ is $\mu$ at a critical point of $E+2 L_{\mu}$. This step works quite generally, yielding a more general existence theory than simply for two-dimensional targets $(N, h)$; we discuss the applications to higher dimensional compact targets and geometrically tame hyperbolic three-manifolds in remarks at the end of $\S 3$.

The difficulty with showing injectivity is that, if $[\sigma],\left[\sigma^{\prime}\right] \in T_{g}$ have corresponding harmonic maps $f(\sigma):(M, \sigma) \rightarrow(N, h)$ and $f\left(\sigma^{\prime}\right):\left(M, \sigma^{\prime}\right) \rightarrow(N, h)$ with equivalent maximal stretch foliations on $M$, we may not be able to detect this equivalence (this equivalence will be defined precisely in $\S 2$ : for the purposes of this introductory section, it suffices to observe that two measured foliations will be equivalent if one is the pullback of the other by an ambient diffeomorphism) by local considerations. In effect, we get past this obstacle by considering the images of the maximal stretch foliations on $N$.

More precisely, associated to a measured foliation on a Riemann surface $\mathcal{R}$ is a dual real tree with an isometric action by $\pi_{1} \mathcal{R}$ : this real tree is obtained by first lifting the measured foliation $(\mathcal{F}, \mu)$ on $\mathcal{R}$ to a measured foliation $(\widetilde{\mathcal{F}}, \tilde{\mu})$ on the universal cover $\widetilde{\mathcal{R}}$, and then projecting (by, say, $p: \widetilde{\mathcal{R}} \rightarrow(T, d)$ ) the universal cover $\widetilde{\mathcal{R}}$ to the leaf space $T$ equipped with the metric $\left(p_{*} \tilde{\mu}\right)$. In the case of a harmonic map $f: \mathcal{R} \rightarrow$ $\left(N^{n}, h\right)$, we consider the maximal stretch measured foliation $(\mathcal{F}, \mu)$, and as noted earlier, then the map $F=(\tilde{f}, p): \widetilde{\mathcal{R}} \rightarrow(\tilde{N}, \tilde{h}) \times(T, 2 d)$ is minimal, and for $N^{2}$ a negatively curved surface, stable. To show uniqueness, we consider an energy function $E^{*}: T_{g} \rightarrow \mathbf{R}$ for equivariant maps of surfaces $(\widetilde{M}, \tilde{\sigma})$ into $(\widetilde{N}, \tilde{h}) \times(T, 2 d)$ which is finite-valued and proper on $T_{g}$. Moreover, $E^{*}$ has critical points only where the Hopf differential has maximal stretch foliation $(\mathcal{F}, \mu)$ with dual tree $(T, d)$; the stability result above then provides that every one of the critical points of $E^{*}$ must be a local minimum. As Teichmüller space is a cell, Morse theory forces a unique minimum.

We organize our discussion as follows. In $\S 2$, we define our terms and notation. In $\S 3$, we state our main result, Theorem 3.1, and begin its proof, showing continuity and surjectivity of the map $\mu: T_{g} \rightarrow M F$. In $\S 4$, we first discuss this construction of minimal maps $F: \widetilde{\mathcal{R}} \rightarrow$ $\left(\widetilde{N}^{n}, \tilde{h}\right) \times(T, 2 d)$ and then prove uniqueness.

The author appreciates useful conversations with H. Masur, Y. Minsky, and B. Tabak, as well as the extraordinarily careful reading and very useful criticism of the referee. 


\section{Definitions and notation}

2.1. Teichmüller space, measured foliations. Let $M$ denote a smooth surface of genus $g \geq 2$, and let $\mathcal{M}_{-1}=\mathcal{M}_{-1}(F)$ denote the space of metrics $\rho|d w|^{2}$ on $M$ with Gaussian curvature identically -1 . The group Diff ${ }_{o}$ of diffeomorphisms of $M$ homotopic to the identity acts on $\mathcal{M}_{-1}$ by pullback: if $\phi \in \operatorname{Diff}_{o}$, then $\phi \cdot \rho=\phi^{*} \rho$. We define the Teichmüller space of genus $g, T_{g}$, to be the quotient space $T_{g}=$ $\mathcal{M}_{-1} /$ Diff $_{o}$, i.e., equivalence classes of metrics in $\mathcal{M}_{-1}$ under the action of Diff ${ }_{o}$.

A measured foliation $(\mathcal{F}, \mu)$ on $M$ is a singular foliation on $M$, where the singularities are isolated and $k$-pronged, equipped with a measure on transverse arcs which is invariant under translation along leaves (see [5] for more details). We define a space $M F(M)$ of equivalence classes of measured foliations by declaring that two measured foliations $(\mathcal{F}, \mu)$ and $\left(\mathcal{F}^{\prime}, \mu^{\prime}\right)$ are equivalent if $\mathcal{F}$ can be taken to $\mathcal{F}^{\prime}$ by a measure preserving isotopy or sequence of Whitehead moves.

2.2. Harmonic maps from surfaces. Let $\left(M, \sigma|d z|^{2}\right)$ and $(N, h)$ denote $M$ and $N$ equipped with smooth Riemannian structures; here $z$ refers to a local conformal coordinate on the surface $M$, and $N$ is an arbitrary $n$-dimensional manifold. For a Lipschitz map $f$ : $\left(M, \sigma|d z|^{2}\right) \rightarrow(N, h)$, we define the energy $E(f ; \sigma, h)$ of the map $w$ to be

$$
\begin{aligned}
E(f ; \sigma, h) & =\int_{M} \frac{1}{2}\|d f\|^{2} d v(\sigma) \\
& =\int_{M} \frac{1}{\sigma(z)}\left\{\left\|f_{*} \partial_{z}\right\|_{h}^{2}+\left\|f_{*} \partial_{\bar{z}}\right\|_{h}^{2}\right\} \sigma(z) d z d \bar{z}
\end{aligned}
$$

Evidently, while the total energy depends upon the metric structure of the target surface $(N, h)$, it only depends upon the conformal structure of the source.

A critical point of this functional is called a harmonic map. We will be interested in the situation where we have fixed a homotopy class $f_{0}: M \rightarrow N$ of maps into the compact target $N$ with all sectional curvatures $K(N)$ satisfying $K(N)<0$. In that case, there is a unique (if $f_{*}\left(\pi_{1} M\right)$ is non-abelian) harmonic map $f(\sigma):(M, \sigma) \rightarrow(N, h)$ in the homotopy class of $f_{0}$; when, in addition, $N$ is two-dimensional and $f_{0}$ is homotopic to a diffeomorphism, we have that $f(\sigma)$ is a diffeomorphism ([4], [11], [21], [18]). 
For harmonic maps $f:(\mathcal{R}, \sigma) \rightarrow(N, h)$ from a Riemann surface $\mathcal{R}$ to a smooth target, one can characterize the harmonicity of $w$ in terms of conformal objects on $\mathcal{R}$. The pullback metric $f^{*} h$ decomposes by type as

$$
\begin{aligned}
f^{*} h= & \left\langle f_{*} \partial_{z}, f_{*} \partial_{z}\right\rangle_{h} d z^{2}+\left(\left\|f_{*} \partial_{z}\right\|_{h}^{2}+\left\|f_{*} \partial_{\bar{z}}\right\|_{h}^{2}\right) \sigma d z d \bar{z} \\
& +\left\langle f_{*} \partial_{\bar{z}}, f_{*} \partial_{\bar{z}}\right\rangle_{h} d \bar{z}^{2} \\
= & \varphi d z^{2}+\sigma e(f) d z d \bar{z}+\bar{\varphi} d \bar{z}^{2}
\end{aligned}
$$

where $e=\frac{1}{2}\|d f\|^{2}$ is the energy density of the map $f$. It is easy to show (see [18]) that if $f$ is harmonic, then $\Phi=\varphi d z^{2}$ is a holomorphic quadratic differential on $\mathcal{R}$. Let $Q D(\mathcal{R})=Q D(\sigma)$ denote the $6 g-6$ dimensional real vector space of holomorphic quadratic differentials on $\mathcal{R}$.

One associates to every holomorphic quadratic differential $\Phi$ on $\mathcal{R}$ a canonical (singular) measured foliation (on the underlying differentiable surface $M$ ) in the following way. If at a point $p \in \mathcal{R}$, we have $\Phi(p) \neq 0$, then by using the canonical coordinate $\zeta(z)=\int_{p}^{z} \sqrt{\Phi(\zeta)} d \zeta$ we write $\Phi=d \zeta^{2}$ in a neighborhood $U \ni p$. If we next write $\zeta=\xi+i \eta$, then the horizontal foliation $\mathcal{F}_{\Phi, \text { hor }}$ is given by the curves $\eta=$ constant, and the transverse measure $\mu_{\Phi, \text { hor }}$ is given locally by setting, for $\gamma \subset$ $U, \mu_{\Phi, \text { hor }}(\gamma)=\int_{\gamma}|d \eta|$. We can also define another measured foliation $\left(\mathcal{F}_{\Phi, \text { ver }}, \mu_{\Phi, \text { ver }}\right)$ similarly using the lines $\{\xi=$ constant $\}$ and $\mu_{\Phi, \text { ver }}=$ $\int_{\gamma}|d \xi|$. These measured foliations easily extend to neighborhoods of the points $p$ where $\Phi(p)=0$; at such points, the foliations have $k$-pronged singularities.

We have described a natural map from $Q D(\mathcal{R})$ into $M F(M)$ which associates to a holomorphic quadratic differential its horizontal measured foliation. Hubbard and Masur [12] (see also Kerckhoff [14], Gardiner [8] and [29] (for an elementary harmonic maps theoretic proof)) showed that this map is a homeomorphism onto all of $M F(M)$.

In the case where $\Phi=d \zeta^{2}$ (locally) is determined as in (2.2) by the harmonic map $f: \mathcal{R} \rightarrow(N, h)$, the foliations $\mathcal{F}_{\Phi, \text { hor }}$ and $\mathcal{F}_{\Phi \text {, ver }}$ integrate the (singular) line fields determined by the maximal and minimal stretch directions of the differential $d f: T \mathcal{R} \rightarrow(T N, h)$.

2.3. R-trees. Let $(T, d)$ be a metric space. There is a natural definition of geodesic in such a space: a geodesic between $t_{0} \in T$ and $t_{1} \in T$ is a path $\gamma_{t_{0} t_{1}}:\left[0, d\left(t_{0}, t_{1}\right)\right] \rightarrow T$ with the property that $d\left(t_{0}, \gamma_{t_{0} t_{1}}(t)\right)=t$. The metric space $(T, d)$ is a real tree if (i) given $t_{0}$, 
$t_{1} \in T$, there is a geodesic $\gamma_{t_{0} t_{1}}$ connecting the point $t_{0}$ to the point $t_{1}$, and (ii) given a triple of points $t_{0}, t_{1}$, and $t_{2}$ in $T$, the geodesics $\gamma_{t_{0} t_{1}}$ and $\gamma_{t_{0} t_{2}}$ agree on $\left[0, \frac{1}{2}\left\{d\left(t_{0}, t_{1}\right)+d\left(t_{0}, t_{2}\right)-d\left(t_{1}, t_{2}\right)\right\}\right]$, i.e., geodesic triangles on $T$ are homeomorphic to a " $Y$ ".

For us, real trees will arise in the following way. Let $(\mathcal{F}, \mu)$ be a measured foliation on the surface $M$, and consider the lifted measured foliation $(\widetilde{\mathcal{F}}, \tilde{\mu})$ on the universal cover $\widetilde{M}$. The leaf space $T$ of the foliation has a natural metric $d_{T}$ defined so that $d_{T}\left(t_{0}, t_{1}\right)=\mu\left(\Gamma_{t_{0} t_{1}}\right)$ where $\Gamma_{t_{0} t_{1}}$ is a quasitransverse arc in $\widetilde{M}$ joining a pre-image of $t_{0}$ to a pre-image of $t_{1}$. It is easy to see that the metric space $(T, d)$ is a real tree. Furthermore, there is a natural projection $p: \widetilde{M} \rightarrow(T, d)$ along the leaves which pushes the transverse measure $\mu$ down to $d$, i.e., $d=p_{*} \mu$; we also observe that the action of the deck group $\pi_{1} M$ on $\widetilde{M}$ descends to an action by isometries on the real tree $(T, d)$, and the map $p$ is $\pi_{1} M$ equivariant.

\section{Main result; continuity, and existence theory for dimensions 2 and higher}

3.1. Our main construction is the following. We begin with a homotopy class of maps determined by $f_{0}: M \rightarrow N$; here $N$ is of course, a surface. Then for each conformal structure $\sigma$ on $M$, there is a unique map $f_{\sigma}:(M, \sigma) \rightarrow(N, h)$ in the homotopy class of $f_{0}$. By pulling back the metric $h$ by $f_{\sigma}$ and decomposing the resulting tensor by type, we obtain a quadratic differential $\Phi(\sigma)=\Phi(\sigma) d z^{2}=\left(f_{\sigma}^{*} h\right)^{2,0} \in Q D(\sigma)$ which is holomorphic with respect to the complex structure $\sigma$. Let $\left(\mathcal{F}_{\sigma}, \mu_{\sigma}\right) \in M F(M)$ denote the horizontal (maximal stretch) foliation of $\Phi(\sigma)$.

This construction amounts to a map $\mu: T_{g} \rightarrow M F(M)$ which assigns to each class of conformal structures $[\sigma] \in T_{g}$ a class of measured foliations $\left(\mathcal{F}_{\sigma}, \mu_{\sigma}\right)$. Our main result is

Theorem 3.1. The map $\mu: T_{g} \rightarrow M F(M)$ is a homeomorphism.

Proof. We argue in three steps: the map $\mu$ is continuous, the map $\mu$ is surjective, and the map $\mu$ is injective.

Step 1. The map $\mu$ is clearly continuous since all of the quantities involved in the construction of $\mu$ vary continuously with $[\sigma] \in T_{g}$ (cf. $[3])$.

Step 2. We claim next that $\mu$ is a surjection. The arguments for 
this step hold in greater generality than simply for 2-dimensional targets. Thus, for this step, we will assume that $N$ is a compact, negatively curved Riemannian manifold, and that $f_{*}$ is an injection on the fundamental group. More general hypotheses are discussed in Remark 3.6.

To see the surjectivity, consider a measured foliation

$$
(\mathcal{F}, \nu) \in M F(M) .
$$

By the Hubbard-Masur theorem [12], we know that given a conformal structure $[\sigma] \in T_{g}$, there is a unique holomorphic quadratic differential $\Psi_{\nu, \sigma} \in Q D(\sigma)$ whose horizontal foliation realizes $(\mathcal{F}, \nu)$. Let $L_{\nu}=$ $\left\|\Psi_{\nu, \sigma}\right\|_{\sigma}$ where $\|\cdot\|_{\sigma}$ denotes the natural $L^{1}$ norm on the Riemann surface $\mathcal{R}=(M, \sigma)$; this construction then exhibits $L_{\nu}$ as a (non-negative) realvalued function $L_{\nu}: T_{g} \rightarrow \mathbf{R}$. Gardiner ([7], [8]) proved that $L_{\nu}$ is differentiable on $T_{g}$ and

$$
d L_{\nu}(\sigma)[\tau]=2 \operatorname{Re} \iint_{M} \Psi_{\nu, \sigma} \tau
$$

here $\tau$ is a Beltrami differential, and $[\tau]$ represents its equivalence class (representing an element of $T_{\sigma} T_{g}$ ). Later, Gardiner and Masur [9] showed that $L_{\nu} \in C^{1}\left(T_{g}\right)$.

Next we consider another $C^{1}$ (non-negative) real-valued function $E: T_{g} \rightarrow \mathbf{R}$ on Teichmüller space given by the energy of a harmonic map. To construct this function, we set $E(\sigma)$ to be the energy of the harmonic map $f_{\sigma}:(M, \sigma) \rightarrow(N, h)$ in the homotopy class of $f_{0}$. Again it is clear [3] that $E \in C^{1}\left(T_{g}\right)$ and we compute

Lemma 3.2. ([19], [24], [25]; see also $[13, \S 6.4])$

$$
d E(\sigma)[\tau]=-4 \operatorname{Re} \iint \Phi(\sigma) \tau .
$$

Remark. The author is unsure as to the precise history of this observation, having first heard of it in lectures of R. Schoen [19] in the winter of 1983 . It also appears in an article [24] of A. Tromba of approximately the same time. Here we provide a proof using the notation and conventions of [8]; it is the sign of derivative which most interests us.

Proof. Consider the energy of a map $u:(M, \sigma) \rightarrow(N, h)$ as a function of two variables $E(\sigma, u)$, the first variable referring to the 
conformal structure and the second variable referring to the map. Then we have

$$
E(\sigma+\epsilon \tau)=E\left(\sigma+\epsilon \tau, f_{\sigma+\epsilon \tau}\right) .
$$

Because the energy of a harmonic map is stationary with respect to variations of the map, we find that

$$
d E(\sigma)[\tau]=\left.\frac{\partial}{\partial \epsilon}\right|_{\epsilon=0} E\left(\sigma+\epsilon \tau, f_{\sigma}\right)
$$

and we compute

$$
\begin{aligned}
\left.\frac{\partial}{\partial \epsilon}\right|_{\epsilon=0} E\left(\sigma+\epsilon \tau, f_{\sigma}\right)= & \left.\frac{\partial}{\partial \epsilon}\right|_{\epsilon=0} \int_{M}\left\langle f_{\sigma_{*}} \frac{\partial}{\partial z_{\epsilon}}, \overline{f_{\sigma_{*}} \frac{\partial}{\partial z_{\epsilon}}}\right\rangle_{h} \\
& +\left\langle f_{\sigma_{*}} \frac{\partial}{\partial \bar{z}_{\epsilon}}, \overline{f_{\sigma_{*}} \frac{\partial}{\partial \bar{z}_{\epsilon}}}\right\rangle_{h} \frac{d z_{\epsilon} \wedge d \bar{z}_{\epsilon}}{2 i},
\end{aligned}
$$

where $z_{\epsilon}$ refers to a local coordinate, conformal with respect to the $\sigma+\epsilon \tau$ structure. From the Beltrami equation $\frac{\partial z_{\epsilon}}{\partial \bar{z}}=\epsilon \tau \frac{\partial z_{\epsilon}}{\partial z}$ we evaluate the right-hand side of the above to

$$
=\iint 2\left\langle f_{\sigma_{*}} \frac{\partial}{\partial z}, \overline{-\bar{\tau} f_{\sigma_{*}} \frac{\partial}{\partial \bar{z}}}\right\rangle+2\left\langle f_{\sigma_{*}} \frac{\partial}{\partial \bar{z}}, \overline{-\tau f_{\sigma_{*}} \frac{\partial}{\partial z}}\right\rangle \frac{d z \wedge d \bar{z}}{2 i}
$$

(where $\frac{\partial}{\partial z}=\frac{\partial}{\partial z_{0}}$ and $\frac{\partial}{\partial z}=\frac{\partial}{\partial z_{0}}$ )

$$
\begin{aligned}
& =-4 \operatorname{Re} \iint\left\langle f_{\sigma_{*}} \frac{\partial}{\partial z}, f_{\sigma_{*}} \frac{\partial}{\partial z}\right\rangle \tau \frac{d z \wedge d \bar{z}}{2 i} \\
& =-4 \operatorname{Re} \iint \Phi(\sigma) \tau \frac{d z \wedge d \bar{z}}{2 i} .
\end{aligned}
$$

Remark 3.3. Inasmuch as the signs of $d L_{\nu}(\sigma)[\tau]$ and $d E(\sigma)[\tau]$ will be important, we offer the following thought-experiment as evidence that the signs should be opposite. We consider a cylinder $C$ described as the planar region $[0, L] \times[0,1]$ with the vertical boundaries $\{0\} \times[0,1]$ and $\{L\} \times[0,1]$ identified. We suppose that there are additional identifications of portions of the boundary components of $C$ so that the identification space is a closed Riemann surface $\mathcal{R}_{0}$. On this surface, we consider the holomorphic quadratic differential $q_{0}$ which is given on $C-\partial C$ as $q_{0}=d z^{2}$ with horizontal measured foliation given in the natural coordinates on $C$ as $(\mathcal{F}, \mu)=(\{y=$ const $\},|d y|)$. We consider deforming $\mathcal{R}_{0}$ to a new Riemann surface $\mathcal{R}_{\epsilon}$ by replacing $[0, L]$ in the 
construction by $[0, L+\epsilon]$; we obtain a new quadratic differential $q_{\epsilon}$. We find then that $L_{\mu}\left(\mathcal{R}_{\epsilon}\right)=L+\epsilon$ so that $\left.\frac{d}{d \epsilon}\right|_{\epsilon=0} L_{\mu}\left(\mathcal{R}_{\epsilon}\right)>0$. On the other hand, if $q_{\epsilon}$ is the Hopf differential of a harmonic map from $\mathcal{R}_{\epsilon}$ to $N$, then in lengthening the maximal stretch leaves $\{y=$ const $\}$ in deforming from $\mathcal{R}_{0}$ to $\mathcal{R}_{\epsilon}$, we have (after a computation which incorporates the change in area form) decreased the portion of energy due to the maximal stretch (horizontal) direction at a cost of a lesser increase in energy due to the minimal stretch (vertical) direction. We conclude that

$$
\left.\frac{d}{d \epsilon}\right|_{\epsilon=0} E\left(\mathcal{R}_{L+\epsilon}\right)<0
$$

Proof of Step 2 (Surjectivity), continued. Consider the function $E+2 L_{\nu}: T_{g} \rightarrow \mathbf{R}$. We observe that this function is $C^{1}$; moreover, because both functions are non-negative, after recalling the Schoen-Yau [22] argument that $E: T_{g} \rightarrow \mathbf{R}$ is a proper function, we find that $E+2 L_{\nu}: T_{g} \rightarrow \mathbf{R}$ is also a proper function. (We sketch the argument of Schoen and Yau: consider a sequence $\left\langle\left[\sigma_{n}\right]\right\rangle$ of points in $T_{g}$ leaving every compact set. If $\left\langle\left[\sigma_{n}\right]>\right.$ also leaves every compact set in the Moduli space $M_{g}$, then using Mumford's [17] observation that the hyperbolic injectivity radius of $\left\langle\left[\sigma_{n}\right]\right\rangle$ is not bounded away from zero, we compute that the energy of the map $f_{\sigma_{n}}:\left(M, \sigma_{n}\right) \rightarrow(N, h)$ must grow arbitrarily large. In the opposite case, where the hyperbolic injectivity radius of $\left\langle\left[\sigma_{n}\right]>\right.$ is bounded away from zero, we use that any uniform bound on $E\left(\sigma_{n}\right)$ would force the maps $f_{\sigma_{n}}$ to be equicontinuous, and thus a subsequence of the surfaces $\left\langle\left[\sigma_{n}\right]\right\rangle$ would converge to an interior point of $T_{g}$.)

Since $E+2 L_{\nu}: T_{g} \rightarrow \mathbf{R}$ is a proper $C^{1}$ function, it must have a critical point in $T_{g}$, that is, a conformal structure $[\sigma]$ with the property that $d\left(E+2 L_{\nu}\right)(\sigma)[\tau]=0$ for every $\tau \in T_{[\sigma]} T_{g}$. Thus, using (3.1) and (3.2) we have

$$
\begin{aligned}
0 & =d\left(E+2 L_{\nu}\right)(\sigma)[\tau] \\
& =-4 \operatorname{Re} \iint \Phi(\sigma) \tau d A(\sigma)+2 \cdot 2 \operatorname{Re} \iint \Psi_{\nu, \sigma} \cdot \tau d A(\sigma) \\
& =-4 \operatorname{Re} \iint\left(\Phi_{\sigma}-\Psi_{\nu, \sigma}\right) \tau d A(\sigma)
\end{aligned}
$$

for every $\tau \in T_{[\sigma]} T_{g}$.

Now $\left(\Phi_{\sigma}-\Psi_{\nu, \sigma}\right)$ is a holomorphic quadratic differential on $(M,[\sigma])$, and (3.3) asserts that $\Phi_{\sigma}-\Psi_{\nu, \sigma}$ is orthogonal (in the natural pairing) to 
every equivalence class of Beltrami differentials. It follows immediately from standard Ahlfors-Bers Teichmüller theory ([2]; see also [8]) that $\Phi_{\sigma}-\Psi_{\sigma, \nu}=0$, or that $\Phi_{\sigma} \equiv \Psi_{\sigma, \nu}$. Thus since $\Psi_{\sigma, \nu}$ has horizontal measured foliation $(\mathcal{F}, \nu)$, we see that the Hopf differential $\Phi_{\sigma}$ for the harmonic map $f_{\sigma}:(M, \sigma) \rightarrow(N, h)$ has maximal stretch (horizontal) measured foliation $(\mathcal{F}, \nu)$.

Remark 3.4. In the case where $(\mathcal{F}, \nu)$ is the zero foliation, we are looking for a conformal harmonic map; the above argument is then precisely the Schoen-Yau ([22]) argument for the existence of a minimal surface in a geometrically finite negatively curved three-manifold.

Remark 3.5. The above proof requires only the properness of the energy functional, and so, in particular, works for any compact target of negative curvature. We conclude

Proposition 3.6. For $(N, h)$ compact and negatively curved and $(\mathcal{F}, \mu) \in M F\left(M_{g}\right)$, in each homotopy class of maps $f_{0}: M_{g} \rightarrow(N, h)$ so that $f_{0 *}$ is injective on $\pi_{1} M$, there exists a conformal structure $[\sigma] \in T_{g}$ such that the maximal stretch foliation of the harmonic map $w(\sigma):\left(M_{g}^{2}, \sigma\right) \rightarrow(N, h)$ is measure equivalent to $(\mathcal{F}, \mu)$.

Remark 3.6. If $(N, h)$ is non-compact, or if $f_{0 *}$ is not injective on $\pi_{1} M$, then the proof continues to hold in some special situations, for instance when $\left(N^{3}, h\right)$ is a convex cocompact hyperbolic three-manifold, because the image of the harmonic maps will meet only the (compact) convex hull. When, $\left(N^{3}, h\right)$ is geometrically infinite but geometrically tame (see, e.g. [31], [32] for definitions and important properties), then we can assert the conclusion of Proposition 3.6 for all measured foliations $(\mathcal{F}, \mu)$ which have non-zero intersection numbers with the relevant ending laminations. This last remark is less immediate, so we indicate an argument. In this case, we can find a family of conformal structures $\left\langle\sigma_{n}\right\rangle$ leaving every compact set in $T_{g}$ and maps $f_{n}:\left(M, \sigma_{n}\right) \rightarrow(N, h)$ so that $E\left(\sigma_{n}, h ; f_{n}\right)$ is uniformly bounded, but the sequence $f_{n}(M)$ leaves every compact set in $N$. Thus, we do not claim that the energy function $E: T_{g} \rightarrow \mathbf{R}$ is proper in this case. Yet, we can consider the entire function $E+2 L_{\nu}: T_{g} \rightarrow \mathbf{R}$ for a fixed measured foliation $(\mathcal{F}, \nu)$ which has non-trivial intersection number $i(\nu, e) \neq 0$ with the ending lamination $e$. We consider a family of conformal structures $\sigma_{n}$ tending to a projective measured foliation $\lambda$ on the Thurston boundary $\partial^{\mathrm{Th}} T_{g}$ of Teichmüller space (see [5], [26] for details and discussion). If $i(\lambda, e) \neq 0$, then one shows (see Minsky [15]) that $E\left(\sigma_{n}, h\right) \rightarrow \infty$. If, on the other 
hand $i(\lambda, e)=0$, then we must have $i(\lambda, \nu) \neq 0$, and one argues that $L_{\nu}\left(\sigma_{n}\right)$ is unbounded. Despite this great abundance of harmonic maps with somewhat controlled geometry, we have no guarantee of the existence of a complex structure whose maximal stretch foliation is trivial, i.e., a minimal surface.

\section{Minimal suspensions and uniqueness}

Step 3. In this section we show that $\mu$ is injective. The proof involves a construction applicable to harmonic maps from surfaces to manifolds of arbitrary dimension which may be of independent interest, and we devote the first subsection to generalities regarding that construction, which we call the minimal suspension of a harmonic map. In the second subsection, we specialize to case of surface targets, where stronger results are possible, and in the third subsection, we apply these special results to prove the injectivity of $\mu$.

4.1. Minimal Suspensions. Let $\mathcal{R}=(M, \sigma)$ denote a Riemann surface $\mathcal{R}$ with the conformal structure $\sigma$, and let $f: \mathcal{R} \rightarrow\left(N^{n}, h\right)$ be a harmonic map from $\mathcal{R}$ to an $n$-manifold $N$. Let $\Phi=\Phi(f, \sigma)$ denote the Hopf differential of this map and let $(\mathcal{F}, \mu)$ denote the maximal stretch measured foliation of $\Phi$.

We can lift this situation to the universal cover $\widetilde{\mathcal{R}}$ of $\mathcal{R}$, finding a $\pi_{1} M$-equivariant lifted map $\tilde{f}: \widetilde{\mathcal{R}} \rightarrow \widetilde{N}$, equivariant Hopf differential $\widetilde{\Phi}$ and equivariant maximal stretch foliation $(\widetilde{\mathcal{F}}, \tilde{\mu})$. As in Section 2.3, if we then project along the leaves of $\widetilde{\mathcal{F}}$, we obtain a real tree $T$, and the transverse measure $\tilde{\mu}$, being invariant under translations along the leaves, projects to a metric $d$ on the tree. The metric space $(T, d)$ is acted upon isometrically by $\pi_{1} M$, and the projection map $p: \widetilde{\mathcal{R}} \rightarrow(T, d)$ is $\pi_{1} M$ equivariant.

Our principal interest is in a scaled tree $(T, 2 d)$ : the effect of the factor of 2 will be to compensate for factors arising in the classical definition of the Hopf differential, as will soon become obvious. Then the projection $p: \widetilde{\mathcal{R}} \rightarrow(T, d)$ is harmonic in either the sense of GromovSchoen [10] or of [28]; in particular, off of the discrete point set $\widetilde{\Phi}^{-1}(0)$, the projection $p$ has the local form $s=p(x, y)=y$ in local coordinates $s$ on $T$ and $z=x+i y$ for which $\widetilde{\Phi}=d z^{2}$ on $\widetilde{\mathcal{R}}$, and is trivially harmonic. The effect of our normalization is that the Hopf differential for $p$ is, in the local coordinates above, $\frac{1}{4}\left(\left\|p_{*} \partial_{x}\right\|^{2}-\left\|p_{*} \partial_{y}\right\|^{2}-2 i\left\langle p_{*} \partial_{x}, p_{*} \partial_{y}\right\rangle\right)=-1$ as $p_{*} \partial_{y}=2 \partial_{s}$ and $p_{*} \partial_{x}=0$ : we conclude that the Hopf differential for 
$p$ is $\left(p^{*} 2 d\right)^{2,0}=-\widetilde{\Phi}$.

This motivates us to form the product space $(\tilde{N}, \tilde{h}) \times(T, 2 d)$ and consider the $\pi_{1} M$ equivariant harmonic map

$$
F=(\tilde{f}, p): \widetilde{\mathcal{R}} \rightarrow(\widetilde{N}, \tilde{h}) \times(T, 2 d) .
$$

We see that the Hopf differential of $F$ is $\left[(f, p)^{*}(\tilde{h}, 2 d)\right]^{2,0}=\widetilde{\Phi}-\widetilde{\Phi}=0$, so that the harmonic map $F$ is also conformal, hence is minimal.

Definition 4.1. We call the equivariant minimal map

$$
F: \widetilde{\mathcal{R}} \rightarrow(\widetilde{N}, \tilde{h}) \times(T, 2 d)
$$

the minimal suspension of $f: \mathcal{R} \rightarrow(N, h)$.

Conversely, we have

Proposition 4.2. Let $F: \widetilde{\mathcal{R}} \rightarrow(\tilde{N}, \tilde{h}) \times(T, 2 d)$ be equivariant, conformal and harmonic and let $\pi_{1}, \pi_{2}$ be the projections of $(\tilde{N}, \tilde{h}) \times(T, 2 d)$ onto the first and second factors, respectively. Then $\pi_{j} \circ F$ is harmonic and the Hopf differential of $\pi_{1} \circ F$ is the negative of the Hopf differential of $\pi_{2} \circ F$. Moreover, the Hopf differential for $\pi_{2} \circ F$ has minimal stretch measured foliation which is equivariantly measure equivalent to $(\widetilde{\mathcal{F}}, \tilde{\mu})$.

Proof. The maps $\pi_{j} \circ F$ are harmonic because $F$ is harmonic and $\pi_{j}$ are isometric submersions. The Hopf differentials are negatives of each other because $F=\left(\pi_{1} \circ F, \pi_{2} \circ F\right): \widetilde{\mathcal{R}} \rightarrow(\tilde{N}, \tilde{h}) \times(T, 2 d)$ is conformal so has zero Hopf differential; we then check that the Hopf differential of a product map is the sum of the Hopf differentials.

To prove that the Hopf differential for $\pi_{2} \circ F$ has minimal stretch foliation which is measure equivalent to $(\widetilde{\mathcal{F}}, \tilde{\mu})$, we proceed as follows. We recall the theorem of Hubbard and Masur [12] which says that on the Riemann surface $\mathcal{R}$, there is a unique holomorphic quadratic differential $\Psi$ whose vertical measured foliation is measure equivalent to $(\mathcal{F}, \mu)$. We lift $\Psi$ to a differential $\widetilde{\Psi}$ on $\widetilde{\mathcal{R}}$ and consider the projection $\pi: \widetilde{\mathcal{R}} \rightarrow$ $(T, 2 d)$ given by projecting along leaves of the vertical foliation of $\widetilde{\Psi}$ : observe that both $\pi$ and $\pi_{2} \circ F$ are equivariant harmonic maps from $\widetilde{\mathcal{R}}$ to $(T, 2 d)$. We consider the function $D(z)=\operatorname{dist}_{(T, 2 d)}\left(\pi_{2} \circ F(z), \pi(z)\right)$; it is not difficult to generalize the result that $D(z)$ is subharmonic from the case of smooth non-positively curved targets to the present case of $(T, 2 d)$ (see [10], [28, Corollary 3.2]). But as $D(z)$ is also equivariant, the function $D(z)$ must achieve its maximum on an interior point, and is therefore a constant. Consider two points $z$ and $z^{\prime}$ on a leaf of the 
vertical foliation of $\widetilde{\Psi}$ connected by a continuous path $A$ within that leaf. Then $\pi(A)$ is a point, and as $\pi_{2} \circ F(A)$ is at a constant distance from the point $\pi(A)$, we have that $\pi_{2} \circ F(A)$ must lie in the totally disconnected subset of the tree of points at a fixed constant distance from $\pi(A)$. We conclude that $\pi_{2} \circ F(A)$ is a point, so that $z$ and $z^{\prime}$ lie along a leaf of the minimal stretch foliation of the Hopf differential for $\pi_{2} \circ F(A)$. Thus the minimal stretch foliation for $\pi_{2} \circ F$ agrees with the vertical stretch foliation of $\widetilde{\Psi}$, i.e., with $\widetilde{\mathcal{F}}$. This forces the germs of the Hopf differential for $\pi_{2} \circ F$ at the zeros to agree with the germs of $\widetilde{\Psi}$ at the zeros of $\widetilde{\Psi}$; the ratio of the Hopf differential of $\pi_{2} \circ F$ to $\widetilde{\Psi}$ is then equivariant, non-vanishing and real at $\widetilde{\Psi}^{-1}(0)$, so we conclude that the Hopf differential of $\pi_{2} \circ F$ is $c \widetilde{\Psi}$ with $c \in \mathbf{R}$. To see that $c=1$, consider the image of a small horizontal arc $B$ of the foliation of $\Psi$. The image of $B$ under $\pi$ is a small arc in $T$ and thus its image under $\pi_{2} \circ F$ is also a small arc translated a constant distance away: since these images are isometric, we see that for a horizontal vector $\partial_{x}$ tangent to $z$ at a point $z \in \widetilde{\mathcal{R}}$, we must have $\left\|\pi_{*} \partial_{x}\right\|=\left\|\pi_{2} \circ F_{*} \partial_{x}\right\|$, so that at that point $z$, we have (in the obvious notation)

$$
\begin{aligned}
\widetilde{\Psi}(z)= & \frac{1}{4}\left(\left\|\pi_{*} \partial_{x}\right\|^{2}-\left\|\pi_{*} \partial_{y}\right\|^{2}-2 i\left\langle\pi_{*} \partial_{x}, \pi_{*} \partial_{y}\right\rangle\right) \\
= & \frac{1}{4}\left\|\pi_{*} \partial_{x}\right\|^{2}=\frac{1}{4}\left\|\left(\pi_{2} \circ F\right)_{*} \partial_{x}\right\|^{2} \\
= & \frac{1}{4}\left(\left\|\left(\pi_{2} \circ F\right)_{*} \partial_{x}\right\|^{2}-\left\|\left(\pi_{2} \circ F\right)_{*} \partial_{y}\right\|^{2}\right. \\
& \left.-2 i\left\langle\left(\pi_{2} \circ F\right)_{*} \partial_{x},\left(\pi_{2} \circ F\right)_{*} \partial_{y}\right\rangle\right) \\
= & \left(\left(\pi_{2} \circ F\right)^{*} d\right)^{2,0}(z) .
\end{aligned}
$$

Thus the Hopf differential for $\pi_{2} \circ F$ is $\widetilde{\Psi}$, which has the prescribed vertical foliation. (Indeed, as the minimal stretch foliation of $\pi_{2} \circ F$ agrees with the vertical stretch foliation of $\widetilde{\Psi}$, we see that the singularities of these foliations coincide. The images of neighborhoods of these singularities in the tree $T$ are a constant distance away, but this is impossible for valence $k \geq 3$ vertices in a negatively curved tree, unless the distance $D=0$. We conclude that $\pi_{2} \circ F=\pi$.) q.e.d.

4.2 Minimal suspensions of maps between surfaces. We now specialize to the case where $N$ is a surface $\left(N^{2}, h\right)$; we will have mild need of the additional assumption that $K_{N}<0$. Thus $\tilde{N} \times T$ is a 3 -dimensional metric space. Our goal in this subsection is to prove 
Theorem 4.3. The minimal suspension $F: \widetilde{\mathcal{R}} \rightarrow \widetilde{N} \times T$ is a stable minimal surface, and strictly stable if the genus of $\mathcal{R}$ is at least 2.

Before we prove the theorem, we need to make a sensible definition of stability for a surface $F(\widetilde{\mathcal{R}})$ in $\widetilde{N} \times T$. To begin we observe that there are two types of local structures for $F$ near a point $q \in \widetilde{\mathcal{R}}$ : if $\widetilde{\Phi}(q) \neq 0$, then for open sets $\mathcal{O} \ni q$ and $V \ni \tilde{f}(q)$, the map $F$ factors as $F=i \circ F_{2}$ where $F_{2}: \mathcal{O} \rightarrow(V, \tilde{h}) \times(-\epsilon, \epsilon)$ is a map into a product Riemannian 3manifold (not merely a three-dimensional metric space) and $i:(V, \tilde{h}) \times$ $(-\epsilon, \epsilon) \rightarrow \widetilde{N} \times(T, 2 d)$ is an isometric inclusion. Indeed, the image $F_{2}(\mathcal{O})$ is a smooth submanifold of $(V, \widetilde{h}) \times(-\epsilon, \epsilon)$, and so possesses a well-defined normal $\vec{n}(q)$ at every point $F_{2}(q)$. Thus, for a deformation $F_{2}^{t}$ of $F_{2}$, one can define an infinitesimal normal displacement $\dot{n}(q)=$ $\left\langle\vec{n}(q),\left.\frac{\partial}{\partial t}\right|_{t=0} F_{2}^{t}(q)\right\rangle$; in the notation we have suppressed the dependence on $F_{2}^{0}$, but this should cause no confusion in our applications. On the other hand, if $\widetilde{\Phi}(q)=0$, then on the open sets $\mathcal{O}$ and $V$, the map $F$ factors as $F=i \circ F_{k}$ where $F_{k}: \mathcal{O} \rightarrow(V, \tilde{h}) \times T^{k}$ with $T^{k}$ being a $k$-pronged star (a bouquet of $k \geq 3$ intervals), and $i$ is an isometric inclusion.

Thus, any compactly supported deformation of $F(\widetilde{\mathcal{R}})$ (not necessarily equivariant) can be taken to lie in a locally compact 3-space, as opposed to the more general (typically) locally non-compact complex $\tilde{N} \times T$.

For our application, as well as for other applications, it suffices to consider deformations of a restricted type. For the purpose of defining these admissable deformations, choose a smooth conformal equivariant metric on $\widetilde{\mathcal{R}}$ and a small positive number $\delta_{0}>0$. Observe that $p\left(B_{\delta_{0}}(q)\right)$ is either an embedded interval in the tree $T$ or a $k$-pronged subtree of the tree $T$. (Here it is important to recognize that $p\left(B_{\delta_{0}}(q)\right.$ ) can be a $k$-pronged subtree without $\widetilde{\Phi}(q)=0$ : one only needs $q$ sufficiently near $\widetilde{\Phi}^{-1}(0)$ for $p\left(B_{\delta_{0}}(q)\right)$ to be more complicated than an embedded interval.)

Recall that $\pi_{2}: \tilde{N} \times T \rightarrow T$ is the projection of $\tilde{N} \times T$ onto the second factor.

Definitions 4.4. a) A deformation $F_{t}:(-\eta, \eta) \times \widetilde{\mathcal{R}} \rightarrow \widetilde{N} \times T$ of a minimal suspension $F: \widetilde{\mathcal{R}} \rightarrow \widetilde{N} \times T$ is strongly admissable if (i) $F_{0}=F$, (ii) $F_{t}(q)$ is continuous in $t$ and $q$, and twice continuously differentiable in $t$ and $q$ off of the $F_{0}$-preimages of the vertices of $T$, (iii) $\pi_{2}\left(F_{t}(q)\right) \in p\left(B_{\delta_{0}}(q)\right)$ and (iv) for $q \notin \Phi^{-1}(0)$ the infinitesimal normal displacement $\dot{n}(q)$ of $F_{t}$ (well-defined by (i), (ii), (iii)) should satisfy 
$\dot{n}(q) \rightarrow 0$ as $q \rightarrow \widetilde{\Phi}^{-1}(0)$.

b) A deformation $F_{t}: \widetilde{\mathcal{R}} \rightarrow \tilde{N} \times T$ of a minimal suspension $F$ : $\widetilde{\mathcal{R}} \rightarrow \widetilde{N} \times T$ is admissable if it satisfies criteria (i), (ii), and (iii) in the definition of strong admissability, as well as $\left(\mathrm{iv}^{\prime}\right)$ the infinitesimal normal displacement $\dot{n}(q)$ satisfies $|\zeta(q)|^{k / 2}|\dot{n}(q)| \rightarrow 0$ as $q \rightarrow q_{0} \in \widetilde{\Phi}^{-1}(0)$ where $k=\operatorname{ord}_{q_{0}} \widetilde{\Phi}$ and $\zeta$ is a local uniformizer at $q_{0}$.

Definition 4.5. The minimal suspension $F$ is (strictly) stable if the second variation of area is (positive) non-negative for every (strongly) admissable equivariant one-parameter deformation $F_{t}: \widetilde{\mathcal{R}} \rightarrow$ $\widetilde{N} \times T$.

Remark 4.6. This definition of strong admissability may seem quite restrictive (and hence the applicability of a stability condition quite narrow), but in fact, we will compute in the next subsection that most of the deformations which most interest us are strongly admissable and the rest are admissable. Moreover, it is easily seen from the computations in the proof of Lemma 4.3 (see (4.2) and (4.3)) that if we did not insist on condition (iv) in the definition of strong admissability, then we will have difficulty defining the infinitesimal normal deformation of $\widetilde{\Phi}^{-1}(0)$ : indeed, in local coordinates $(x, y, s)$ for $(V, \tilde{h}) \times(-\epsilon, \epsilon)$, the normal to $F_{2}$ has the expression

$$
\vec{n}=\frac{-2\left(1+|\tau|^{2}\right)}{\left(1-|\tau|^{2}\right)} \frac{\partial}{\partial y}+\frac{1}{2} \frac{1-|\tau|}{1+|\tau|} \frac{\partial}{\partial s},
$$

where $\tau$ represents the Beltrami differential of the harmonic map $\tilde{f}:(\widetilde{M}, \sigma) \rightarrow(\widetilde{N}, \tilde{h})$. At a zero $q$ of the Hopf differential $\widetilde{\Phi}$, we have $|\tau|(q)=0$. So consider one of the prongs $\underline{P}$ of the maximal stretch foliation $\widetilde{\mathcal{F}}$ emanating from a zero $q$ of $\widetilde{\Phi}$, and observe that neighborhoods of points on this prong map to a well-defined arc $A_{\underline{P}}$ through the $k$-pronged star $p\left(B_{\delta_{0}}(q)\right)$. But these normals along the prong $\underline{P}$ all have a component in the direction of (the image of) $A_{\underline{P}}$ (in $\widetilde{N} \times T$ ), and that component does not vanish as $q^{\prime} \in \underline{P}$ tends to $q \in \widetilde{\Phi}^{-1}(0)$. But this means that there is no consistent choice of normal at $q$, as limits along different prongs $\underline{P}$ evidently lead to different components along the tree direction $\widetilde{N} \times T$.

On the other hand, we will find that the weaker condition $\left(\mathrm{iv}^{\prime}\right)$ in the definition of (non-strong) admissability will suffice to control the contribution to the second order change in area of a neighborhood near $\widetilde{\Phi}^{-1}(0)$ where a normal vector $\dot{n}$ is not consistently defined: after an integration by parts, the stability integral will involve a damping factor 
of order $|\zeta(q)|^{k-1}$ (where $q \rightarrow q_{0} \in \widetilde{\Phi}^{-1}(0), k=\operatorname{ord}_{q_{0}} \widetilde{\Phi}$ and $\zeta$ is a local uniformizer near $\left.q_{0}\right)$ on the boundary of a small $|\zeta|$-disk around $q_{0}$.

Our plan is to compute this stability integrand in a neighborhood $V \subset \widetilde{\mathcal{R}}$ away from $\widetilde{\Phi}^{-1}(0)$. We choose coordinates $z=x+i y$ so that $\widetilde{\Phi}(z)=d z^{2}$ : this forces the maximal stretch foliation to be $\{y=$ const $\}$ with transverse measure $|d y|$. In those coordinates, we compute that the metric on $\widetilde{N}$ in the image of this neighborhood is $d s_{(\widetilde{N}, \tilde{h})}^{2}=(\tilde{\sigma} e+$ 2) $d x^{2}+(\tilde{\sigma} e-2) d y^{2}$ where $e$ denotes the energy density of the map $\tilde{f}:(\widetilde{M}, \tilde{\sigma}) \rightarrow(\tilde{N}, \tilde{h})$. Thus, we further compute (in coordinates $(u, v, s)$ of the image $F(V))$ that

$$
\begin{aligned}
& d s_{(\tilde{N}, \tilde{h}) \times(T, 2 d)}^{2}=(\tilde{\sigma} e+2) d u^{2}+(\tilde{\sigma} e-2) d v^{2}+4 d s^{2}, \\
& F(x, y)=F_{2}(x, y)=(x, y, y), \\
& \vec{n}=\left(0, \frac{-2}{\sqrt{(\tilde{\sigma} e-2)(\tilde{\sigma} e+2)}}, \frac{1}{2} \sqrt{\frac{\tilde{\sigma} e-2}{\tilde{\sigma} e+2}}\right),
\end{aligned}
$$

where $\vec{n}$ is the normal to the image $F(\widetilde{\mathcal{R}})$, and from (4.1) and (4.2) that the induced metric on the target is

$$
\gamma=d s_{F(\tilde{\mathcal{R}})}^{2}=(\tilde{\sigma} e+2)|d z|^{2} .
$$

Proof of Theorem 4.3. In view of Definition 4.5, it is enough to consider deformations expressed as $F_{t}(z)=F(z)+t g(z) \vec{n}+0\left(t^{2}\right)$ where $g(z)$ vanishes on $\Phi^{-1}(0)$. Thus, we may use the classical stability condition; it is convenient to use the formulation of Schoen-Yau [22, Proof of Theorem 5.1]

$$
\int_{F(\tilde{\mathcal{R}})}\left|\nabla_{\gamma} g\right|^{2}-\left(R-K+\frac{1}{2} \Sigma h_{i j}^{2}\right) g^{2} d A_{\gamma} \geq 0
$$

Here $\nabla_{\gamma}$ refers to the gradient with respect to the induced metric $\gamma$ on the target $F(\widetilde{\mathcal{R}})$, the term $R$ represents the scalar curvature of $\widetilde{N} \times T$, the term $K$ represents the Gaussian curvature of $F(\widetilde{\mathcal{R}})$, the form $d A_{\gamma}$ is the area form for the metric $\gamma$, and $\left(h_{i j}\right)$ is the second fundamental form of the minimal surface $F(\widetilde{\mathcal{R}})$ (so that $\left(h_{i j}\right)$ is symmetric and traceless).

It is then straightforward to compute the Christoffel symbols

$$
\Gamma_{b c}^{a}=\frac{1}{2} g^{p a}\left(\partial_{c} g_{b p}+\partial_{b} g_{c p}-\partial_{p} g_{b c}\right)
$$


for the metric $d s_{\tilde{N} \times T}^{2}=g_{a b} d u^{a} d u^{b}$ above, as well as the components of the second fundamental form $h_{i j}=\left\langle\nabla_{X^{i}}^{N \times T} \vec{n}, X^{j}\right\rangle(i, j=1,2)$, where we set $X^{1}=\frac{1}{\sqrt{\sigma e+2}} \partial_{x}$ and $X^{2}=\frac{1}{\sqrt{\sigma e+2}}\left(\partial_{y}+\partial_{s}\right)$ on $F(\widetilde{\mathcal{R}})$. Our next computations being local, we will suppress much of the notation designed to indicate lifts to universal covers.

We find that

$$
\begin{aligned}
& h_{11}=-h_{22}=-\frac{1}{(\sigma e-2)^{1 / 2}(\sigma e+2)^{3 / 2}}\left(\partial_{y}(\sigma e+2)\right), \\
& h_{12}=h_{21}=\frac{1}{(\sigma e-2)^{1 / 2}(\sigma e+2)^{3 / 2}}\left(\partial_{x}(\sigma e+2)\right) .
\end{aligned}
$$

Thus

$$
\sum_{i, j=1}^{2} h_{i j}^{2}=\frac{2}{(\sigma e+2)^{3}(\sigma e-2)}\left|\nabla_{0} \sigma e\right|^{2}
$$

where $\nabla_{0}$ refers to the gradient with respect to the Euclidean metric $|\Phi|=|d z|^{2}$. Now, on our neighborhood, the map $F$ factors as $i \circ F_{2}$, where $i$ is an isometric embedding, as in the discussion preceeding Definitions 4.4. Thus near the $F_{2}$-image of our neighborhood, the range may be represented as a product manifold $\widetilde{N} \times(-\epsilon, \epsilon)$, so that the scalar curvature is $K_{\tilde{N}}$ : we conclude that in our coordinates

$$
\begin{aligned}
R=K_{\tilde{N}}= & -\frac{1}{2} \frac{1}{(\sigma e-2)(\sigma e+2)}\left(\partial_{x}^{2}+\partial_{y}^{2}\right)(\sigma e) \\
& +\frac{(\sigma e)\left|\nabla_{0}(\sigma e)\right|^{2}}{2(\sigma e+2)^{2}(\sigma e-2)^{2}} .
\end{aligned}
$$

Finally, given the information in (4.4), it is easy to find that

$$
K_{F(\tilde{\mathcal{R}})}=-\frac{1}{2} \frac{1}{(\sigma e+2)^{2}}\left(\partial_{x}^{2}+\partial_{y}^{2}\right)(\sigma e)+\frac{\left|\nabla_{0} \sigma e\right|^{2}}{2(\sigma e+2)^{3}} .
$$

Combining (4.6), (4.7) and (4.8), we find

$$
\begin{aligned}
R-K+\frac{1}{2} \sum_{i, j=1}^{2} h_{i j}^{2}= & \frac{-2}{(\sigma e+2)^{2}(\sigma e-2)}\left(\partial_{x}^{2}+\partial_{y}^{2}\right)(\sigma e) \\
& +\frac{(4 \sigma e-4)}{(\sigma e+2)^{3}(\sigma e-2)^{2}}\left|\nabla_{0} \sigma e\right|^{2} .
\end{aligned}
$$


Rewriting the second order terms yields

$$
\begin{aligned}
R-K+\frac{1}{2} \sum_{i, j=1}^{2} h_{i j}^{2}= & \operatorname{div}_{0}\left[\frac{-2 \nabla_{0}(\sigma e)}{(\sigma e+2)^{2}(\sigma e-2)}\right]+\frac{(-6 \sigma e+4)\left|\nabla_{0} \sigma e\right|^{2}}{(\sigma e+2)^{3}(\sigma e-2)^{2}} \\
& +\frac{(4 \sigma e-4)\left|\nabla_{0} \sigma e\right|^{2}}{(\sigma e+2)^{3}(\sigma e-2)^{2}} \\
= & \operatorname{div}_{0}\left[\frac{-2 \nabla_{0}(\sigma e)}{(\sigma e+2)^{2}(\sigma e-2)}\right]-\frac{2 \sigma e\left|\nabla_{0} \sigma e\right|^{2}}{(\sigma e+2)^{3}(\sigma e-2)^{2}} .
\end{aligned}
$$

We are therefore integrating the form

$$
\begin{aligned}
& \left\{\left\|\nabla_{\gamma} g\right\|_{F(\widetilde{\mathcal{R}})}^{2}-\left(R-K+\frac{1}{2} \sum_{i, j=1}^{2} h_{i j}^{2}\right) g^{2}\right\} d A_{\gamma} \\
& =\left\{\frac{\left|\nabla_{0} g\right|_{0}^{2}}{\sigma e+2}-\left(\operatorname{div}_{0}\left[\frac{-2 \nabla_{0}(\sigma e)}{(\sigma e+2)^{2}(\sigma e-2)}\right]\right) g^{2}\right. \\
& \left.+\frac{2 \sigma e\left|\nabla_{0} \sigma e\right|^{2} g^{2}}{(\sigma e+2)^{3}(\sigma e-2)^{2}}\right\}(\sigma e+2) d x d y \\
& =\left\{\left|\nabla_{0} g\right|^{2}-\operatorname{div}_{0}\left[\frac{-2\left(\nabla_{0}(\sigma e)\right) g^{2}(\sigma e+2)}{(\sigma e+2)^{2}(\sigma e-2)}\right]\right. \\
& -\frac{2 \nabla_{0}(\sigma e) \cdot \nabla_{0}\left(g^{2}(\sigma e+2)\right)}{(\sigma e+2)^{2}(\sigma e-2)} \\
& \left.+\frac{2 \sigma e(\sigma e+2)\left|\nabla_{0} \sigma e\right|^{2} g^{2}}{(\sigma e+2)^{3}(\sigma e-2)^{2}}\right\} d x d y \\
& =\left\{\left|\nabla_{0} g-\frac{2 g \nabla_{0} \sigma e}{(\sigma e+2)(\sigma e-2)}\right|^{2}+\operatorname{div}_{0}\left[\frac{2 g^{2} \nabla_{0}(\sigma e)}{(\sigma e+2)(\sigma e-2)}\right]\right\} d x d y .
\end{aligned}
$$

We rewrite the terms more invariantly; first observe that

$$
\begin{aligned}
\frac{\nabla_{0}(\sigma e)}{(\sigma e+2)(\sigma e-2)} & =\nabla_{0} \log \frac{\sigma e-2}{(\sigma e+2)} \\
& =\nabla_{0} \log \frac{e-\frac{2|\Phi|}{\sigma}}{e+\frac{2|\Phi|}{\sigma}} \\
& =2 \nabla_{0} \log \frac{1-|\tau|}{1+|\tau|},
\end{aligned}
$$

where we are using that $\tau=\frac{f_{\bar{z}}}{f_{z}}, \Phi=\sigma \mathcal{H} \bar{\tau}$, and $e=\mathcal{H}+\mathcal{L}$ where $\mathcal{H}=\sigma \frac{1}{2}\left\|f_{z}\right\|_{h}^{2}$ and $\mathcal{L}=\sigma \frac{1}{2}\left\|f_{\bar{z}}\right\|_{h}^{2}$. 
Thus, we conclude

$$
\left\{\left\|\nabla_{\gamma} g\right\|_{F(\tilde{\mathcal{R}})}^{2}-\left(R-K+\frac{1}{2} \sum_{i, j=1}^{2} h_{i j}^{2}\right) g^{2}\right\} d A_{\gamma}
$$

$$
=\left\{\left|\nabla_{0} g-4 g \nabla_{0} \log \frac{1-|\tau|}{1+|\tau|}\right|^{2}+\operatorname{div}_{0}\left[4 g^{2} \nabla_{0} \log \frac{1-|\tau|}{1+|\tau|}\right]\right\} d x d y .
$$

It is now straightforward to argue from (4.9) and (4.5) that a minimal suspension is stable. First observe that as $z \rightarrow \widetilde{\Phi}^{-1}(0)$, we have both $|\tau|=|\widetilde{\Phi}| / \sigma \mathcal{H}=O\left(|z|^{k}\right)$ and by admissability, $g(z)^{2}=o\left(|z|^{k}\right)$. Thus, $g^{2} \nabla_{0} \log \frac{1-|\tau|}{1+|\tau|}=o\left(|z|^{-1}\right)$, and so we can integrate (4.9) over the complement in $\widetilde{\mathcal{R}} / \Gamma$ of small $\kappa$-neighborhoods of $\widetilde{\Phi}^{-1}(0)$ and obtain a non-negative term from the first term in (4.9) and a boundary term that vanishes as $\kappa \rightarrow 0$. We conclude that $F$ is stable.

Next we claim that if the genus of $\mathcal{R}$ is at least 2 and the deformation strongly admissable, then the map $F$ is strictly stable. To see this we observe that if $F$ were stable but not strictly stable then we would have, locally,

$$
\nabla_{0} g=4 g \cdot \nabla_{0} \log \frac{1-|\tau|}{1+|\tau|}
$$

But then this forces

$$
g=c\left[\frac{1-|\tau|}{1+|\tau|}\right]^{4}
$$

for a non-zero constant $c$. Thus, if $\Phi(q)=0$, we have that $|\tau|(q)=0$ so that $g(q) \neq 0$. This contradicts the admissability of the deformation, so that we must have strict inequality in (4.5) unless $\Phi$ has no zeros. But, of course, as soon as the genus of $\widetilde{\mathcal{R}}$ is at least 2, the holomorphic quadratic differential must have zeros, by the Riemann-Roch Theorem. q.e.d.

For some later discussion (in Section 4.3.2) concerning deformations in which high order zeros decay into several lower order zeros, we record a more technical version of the last paragraph of the above proof.

Corollary 4.7. For every admissable equivariant one-parameter deformation $F_{t}: \widetilde{\mathcal{R}} \rightarrow N \times T$ of a minimal suspension $F: \widetilde{\mathcal{R}} \rightarrow N \times T$, the second variation of area is positive unless $\dot{n}=c((1-|\tau|) /(1+|\tau|))^{4}$. 
Remarks 4.8. a) When $\mathcal{R}$ is a torus and $N$ a flat torus, the situation is very simple. The harmonic map $f$ is an affine stretch, and the minimal and maximal stretch foliations are given by parallel geodesics (usually open) on both $\mathcal{R}$ (with the flat metric) and $N$. Consider, at a point $q \in \mathcal{R}$, a vector $v_{m}$ tangent to the minimal stretch foliation and an orthogonal vector $v_{M}$ of the same length tangent to the maximal stretch foliation. The harmonic map takes these vectors to orthogonal vectors on $N$ of different lengths. Thus, to make the map conformal, it would suffice to stretch the minimal stretch foliation so that the image of $v_{m}$ had the same length as the image of $v_{M}$. This is the effect of the minimal suspension, which in this case merely stretches the minimal stretch foliation by the appropriate amount by forcing the minimal stretch leaves to incline at a slope of $\left(\left\|f_{*} v_{M}\right\|^{2}-\left\|f_{*} v_{m}\right\|^{2}\right)^{1 / 2} /\left\|f_{*} v_{M}\right\|$.

b) Of course, to apply Theorem 4.3, we need to show that a particular deformation family which we are considering is admissable. In our proof of uniqueness, this family will be a certain family of maps induced by a path in Teichmüller space: the main technical result of the next subsection is to show that this deformation family is admissable.

4.3. Proof of uniqueness. Our plan for uniqueness is now the following. Let $\mathcal{R}=(M, \sigma)$ denote a Riemann surface whose associated maximal measured foliation, in the sense of Section 3 , is $(\mathcal{F}, \mu)$, and let $(T, 2 d)$ denote the $\mathbf{R}$-tree associated to $(\mathcal{F}, \mu)$, in the sense of Subsections 2.3 and 4.1. We will consider, for each element $[\rho]$ in the Teichmüller space $T_{g}$, a map $F_{\rho}:(\widetilde{M}, \widetilde{\rho}) \rightarrow(\widetilde{N}, \tilde{h}) \times(T, 2 d)$. The images $F_{\rho}(\widetilde{M})$ are a family of surfaces, parametrized by Teichmüller space. Thus a path $\left[\rho_{t}\right] \subset T_{g}$ through $\left[\rho_{0}\right]=[\sigma] \in T_{g}$ determines a deformation of the minimal suspension $F_{\rho_{0}}=F_{\sigma}: \widetilde{\mathcal{R}} \rightarrow \widetilde{N} \times T$. Our main technical goal in this section is to appropriately parametrize such a neighborhood of Teichmüller space so that this deformation family is admissable or strongly admissable, in the sense of Definitions 4.4. Then Theorem 4.3 (and in a non-generic case, Corollary 4.7) will say that the (equivariant) area of $F_{\rho}$ has a unique local minimum at $[\rho]=[\sigma]$. We then consider an associated equivariant energy function $E[\rho]=E\left[F_{\rho}\right]$ which will then also have local minimum in $T_{g}$ only at those conformal structures $[\rho]$ for which the maximal stretch measured foliation of $\Phi_{\rho}$ is $(\mathcal{F}, \mu)$. Uniqueness will thus follow from an appropriate Morse theory argument by using that Teichmüller space is a cell.

4.3.1. To begin, consider a point $[\rho]$ in $T_{g}$. We know that for each 
such point, there is a unique harmonic map $f_{\rho}:(M, \rho) \rightarrow(N, h)$ in the prescribed homotopy class and a holomorphic quadratic differential $\Psi_{\rho}=\Psi_{\mu, \rho} \in \mathrm{QD}(\rho)$ whose horizontal measured foliation is $(\mathcal{F}, \mu)$; we see that the projection $p_{\rho}:(\widetilde{M}, \tilde{\rho}) \rightarrow(T, 2 d)$ along the horizontal leaves of the lift $\widetilde{\Psi}_{\rho}$ is harmonic. Thus we can look at the product map $F_{\rho}=$ $\left(\tilde{f}_{\rho}, p_{\rho}\right):(\widetilde{M}, \widetilde{\rho}) \rightarrow(\widetilde{N}, \tilde{h}) \times(T, 2 d)$. As before this map is harmonic, although, in general, it may not be conformal. Still, we can measure the equivariant energy $E^{*}$ of the product map,

$$
E^{*}=\frac{1}{2} \iint_{\widetilde{M} / \pi_{1} M} \sum_{j}\left\|F_{\rho *} e_{j}\right\|^{2} d A_{\tilde{\rho}},
$$

(in the natural notation) which we can easily compute to be the function $E^{*}=E+2 L_{\mu}$ of $\S 3$. We summarize properties of this construction in

Proposition 4.9. $E^{*}: T_{g} \rightarrow \mathbf{R}$ is a $C^{1}$ function on $T_{g}$, and at critical points $\rho_{0} \in T_{g}$ of $E^{*}$, we have that $F_{\rho_{0}}$ is minimal and $E^{*}=$ $A\left(F_{\rho_{0}}\right)$, where $A\left(F_{\rho_{0}}\right)$ denotes the equivariant area. For general $[\rho] \in T_{g}$, $E^{*}([\rho]) \geq A\left(F_{\rho}\right)$. Finally $E^{*}$ is a proper function on $T_{g}$.

Proof. The first statement is due to Gardiner and Masur [9] using that $E^{*}=E+2 L \mu$; formula (3.3) and the paragraph following it show that $F_{\rho_{0}}$ is conformal, hence minimal. The conformality of $F_{\rho_{0}}$ shows that $E^{*}=A\left(F_{\rho_{0}}\right)$. The general inequality $E^{*}([\rho]) \geq A\left(F_{\rho}\right)$ follows from the arithmetic-geometric inequality. Finally, observe that the projection $\pi_{1} \circ F_{\rho}:(\widetilde{M}, \widetilde{\rho}) \rightarrow(\widetilde{N}, \tilde{h})$ is the product of a harmonic map with an isometric submersion, and consequently reduces the energy density. Of course, $\pi_{1} \circ F_{\rho}=\tilde{f}_{\rho}$, and so $E^{*} \geq E\left(f_{\rho}\right)$; however, Schoen-Yau [22] show that $E\left(f_{\rho}\right): T_{g} \rightarrow \mathbf{R}$ is a proper function on $T_{g}$, so that $E^{*}$ is also. q.e.d.

Our next goal is a proof of the uniqueness theorem; this will require several steps in two cases.

4.3.2. Case 1. We consider first the (generic) case where $(\mathcal{F}, \mu)$ has only simple singularities which are not connected by leaves of the foliation. We begin with

Lemma 4.10. The family of equivariant harmonic maps $F_{\rho_{t}}$ : $\left(\widetilde{M}, \tilde{\rho}_{t}\right) \rightarrow(\widetilde{N}, \tilde{h}) \times(T, 2 d)$ depending on a parameter $t \in(-\epsilon, \epsilon)$ and where $\rho_{0}$ is critical for $E^{*}: T_{g} \rightarrow \mathbf{R}$ induces a deformation of $F_{\rho_{0}}$ which is strongly admissable in the sense of Definition 4.4, i.e., has a trivial 
normal displacement at $\widetilde{\Psi}_{\widetilde{\rho}_{0}}^{-1}(0)$. Here $T$ is the tree associated to $(\mathcal{F}, \mu)$, and $(\mathcal{F}, \mu)$ has only simple zeros, unconnected by leaves of $\mathcal{F}$.

Proof. We summarize the situation as follows. The family $F_{\rho_{t}}=$ $\left(\tilde{w}_{t}, p_{t}\right)$ is the product map of a harmonic map $\tilde{w}_{t}:\left(\widetilde{M}, \tilde{\rho}_{t}\right) \rightarrow(\widetilde{N}, \tilde{h})$ from $\left(\widetilde{M}, \tilde{\rho}_{t}\right)$ to $(\widetilde{N}, \tilde{h})$ and the harmonic map $p_{t}:\left(\widetilde{M}, \tilde{\rho}_{t}\right) \rightarrow(T, 2 d)$ from $\left(\widetilde{M}, \tilde{\rho}_{t}\right)$ to $(T, 2 d)$ which has the effect of realizing the foliation $(\mathcal{F}, \mu)$ on $\left(\widetilde{M}, \tilde{\rho}_{t}\right)$, i.e., via the holomorphic quadratic differential $\widetilde{\Psi}_{t}$. Now, while the actual harmonic map $\tilde{w}_{t}$ depends upon the structure $\tilde{\rho}_{t}$ (i.e., upon the choice of representative in the Diff ${ }^{\text {-equivalence class }}$ $\left.\left[\tilde{\rho}_{t}\right]\right)$, the image $\tilde{w}_{t}(\widetilde{\Psi})$ in $N$ depends only on the class $\tilde{\rho} \in\left[\tilde{\rho}_{t}\right]$. Thus, in analyzing the image $F_{\rho t}(M)$ in $\widetilde{N} \times T$, and in particular in estimating a deformation away from $F_{\rho_{0}}$ near $\widetilde{\Psi}_{\rho_{0}}^{-1}(0)$, we are interested in estimating the deformation of the image under $\tilde{w}_{t}$ of the horizontal foliation of $\widetilde{\Psi}_{t}$. In fact, the lemma only discusses the deformation of these images in a neighborhood of $\widetilde{\Psi}_{\rho_{0}}^{-1}(0)$.

For the sake of clarity, we break the argument up into several steps. At first we will consider a family of metrics $\rho_{t}$ (and associated conformal structures $\left[\rho_{t}\right]$ ) on the fixed differentiable surface $M$ where the metrics depend differentiably on the parameter $t \in(-\epsilon, \epsilon)$. We will assume that the zero set $\Psi_{t}^{-1}(0)$ is also differentiable in $t$. Then under this assumption we will consider a point $\zeta \notin \Psi^{-1}(0)$ and (i) estimate from below the $h$-distance $d_{h}\left(w_{t}(\zeta), w_{t}\left(\Psi^{-1}(0)\right)\right.$ from $w_{t}(\zeta)$ to $w_{t}\left(\Psi^{-1}(0)\right)$ and (ii) estimate from above the $t$-derivative

$$
\left.\frac{d}{d t}\right|_{t=0} d_{(T, 2 d)}\left(p_{t}(\zeta), p_{t}\left(\Psi^{-1}(0)\right)\right)
$$

Both of these estimates will be in terms of the value $\zeta$. Of course, we are ultimately interested in determining the size $|\dot{n}(\zeta)|$ of the normal displacement $\dot{n}(\zeta)$ as a function of $|\zeta|$. But formula (4.3) can be read to assert that the $|\dot{n}(\zeta)|$ is comparable near $\widetilde{\Phi}^{-1}(0)$ to either its $y$ - or its $s$-coordinates, and (4.12) is precisely the variation of (each of) these coordinates. Then when we compare the results of the estimates (i) and (ii) above, we will find that $|\dot{n}(\zeta)|=o\left(d_{h}\left(w_{0}(\zeta), w_{0}\left(\Psi^{-1}(0)\right)\right)\right.$. This is precisely the requirement for strong admissability in Definition 4.4.

Our second step will be to verify the assumption that the zero set $\Psi_{t}^{-1}(0)$ varies differentiably in $t$ on $M$.

Remarks 4.11. (i) It is mostly in this second step that we will need to alter the argument for the case that $\tilde{\Phi}$ has non-simple zeros: the rest of the argument will require only modest changes. 
(ii) Let us observe that $\dot{n}(\zeta)$ is well-defined for $\zeta \notin \widetilde{\Phi}^{-1}(0)$, even when the zeroes are not simple. This is so because for $\zeta \notin \Phi^{-1}(0)$, we can find an interval of time $(-\eta(\zeta), \eta(\zeta))$ and a radius $\delta(\zeta)$ so that for $t \in(-\eta(\zeta), \eta(\zeta))$ we have $p_{t}(\zeta) \in p_{0}\left(B_{\delta(\zeta)}(\zeta)\right)$, i.e., the image of $\zeta$ under $p_{t}$ varies only in the original prong of the subtree $p\left(B_{\delta_{0}}(\zeta)\right)$, in the notation of the paragraph preceeding Definitions 4.4 and 4.5. Thus, we can define $\left.\frac{d}{d t}\right|_{t=0} F_{\rho_{t}}(\zeta)$; note here that we do not need to find a uniform interval of time $(-\eta, \eta)$ to define the normal $\dot{n}(\zeta)$. We will see in the next lemma that this is also sufficient information to conclude from strict stability that $E^{*}\left(F_{\rho_{t}}\right)>E\left(F_{\rho_{0}}\right)$.

(iii) In the first paragraph of the proof, we pointed out that while the harmonic map $\tilde{w}_{t}$ depends on the conformal structure $\tilde{\rho}_{t}$, and its coordinate expressions depend on the choice of conformal coordinate $z_{t}$, the image $\tilde{w}_{t}(\widetilde{\Psi})$ is well-defined. Nevertheless, in carrying out our plan. it is much more convenient to compute in coordinates in the domain $M$ than it would be to compute in range coordinates; we will therefore compute in the domain coordinates, carefully normalizing these coordinates as we go so that no substantive contributions are made from the choice of families of coordinates.

We now carry out the plan described above. We consider a point $\zeta$ in a coordinate disk near $q \in \widetilde{\Phi}^{-1}(0)$, bearing in mind its Diff -invariant $^{-}$ interpretation as $\tilde{w}_{t}(\zeta)$; we will normalize our conformal coordinates $z_{t}$ so that $z_{t}(q)=0$ and $\left|z_{t}(\zeta)\right|$ is both comparable to $d_{w^{*} h}\left(z_{0}(\zeta), z_{0}(q)\right)$ and has $\left|\frac{d}{d t} z_{t}(\zeta)\right|$ bounded. Then since $\tilde{w}_{t}$ is conformal at $\zeta$, and uniformly quasiconformal on $\widetilde{M}$, we see that

$$
d_{\tilde{h}}\left(\tilde{w}_{0}(\zeta), \tilde{w}_{0}(q)\right) \geq c\left[e\left(\tilde{w}_{0}\right)(q)\right]|\zeta| \geq c_{1}|\zeta|
$$

Thus, coordinate patch distances are comparable (an analogous argument for the opposite inequality is clear) to $\widetilde{N}$-distances.

Next, we undertake the critical estimate of the variation of $F_{\rho_{t}}(\zeta)$. For this, it is sufficient to compute (4.12). So we write

$$
\Psi_{t}=\left(z_{t}-a(t)\right) d z_{t}^{2}
$$

in the (previously normalized) conformal coordinate $z_{t}$; here $\Psi_{t}$ has a simple zero at $a(t)$, and we take $a(t)$ to be differentiable in $t$ (per our initial italicized assumption to be justified later) and $a(0)=0$. All of these considerations take place in a fixed open set in $M$. Hence, in our 
coordinates

$$
\begin{aligned}
\left\|\pi_{2 *}\left[\left.\frac{d}{d t}\right|_{t=0} F_{\rho t}(\zeta)\right]\right\| & =\left|\frac{d}{d t}\right|_{t=0} d_{(T, 2 d)}\left(p_{t}(\zeta), p_{t}\left(\Psi_{t}^{-1}(0)\right)\right) \mid \\
& =\left|\frac{d}{d t}\right|_{t=0} \int_{a(t)}^{\zeta} \operatorname{Im} \sqrt{\Psi_{t}} \mid \\
& =\left|\frac{d}{d t}\right|_{t=0} \int_{a(t)}^{\zeta} \operatorname{Im} \sqrt{z_{t}-a(t)} d z_{t} \mid \\
& =O\left(|\zeta|^{1 / 2}\right) \frac{d}{d t} a(t) .
\end{aligned}
$$

It is thus apparent that $\dot{n}(\zeta)=o\left(d_{\tilde{h}}\left(\tilde{w}_{0}(\zeta), \tilde{w}_{0}\left(\Psi_{0}^{-1}(0)\right)\right)\right.$, i.e., $\dot{n}(\zeta)=$ $o(|\zeta|)$ as required by the definition of strong admissability, as long as $\dot{a}(\zeta)$ is bounded.

The second part of our plan was to show that $\dot{a}(\zeta)$ is bounded in this chart, or in a chart differentiably near this chart. Now, to do this, we need only work on the compact Riemann surface instead of on the universal cover. Indeed, the harmonic map to $(N, h)$ provides a canonical section of $\mathcal{M}_{-1} \rightarrow T_{g}$, i.e., we choose a conformal structure $\rho_{t}$ on $M$ so that the identity map id $:\left(M, \rho_{t}\right) \rightarrow(N, h)=(M, \rho)$ is harmonic.

Then choose some family of curves $\left(\gamma_{1}, \ldots, \gamma_{6 g-6}\right)$ which (i) are transverse to the horizontal foliation of $\Phi$, (ii) avoid neighborhoods of $\Phi^{-1}(0)$, and (iii) are in free homotopy classes whose transverse measures provide local coordinates for $\mathcal{M F}$ near $[(\mathcal{F}, \mu)]$. Let $Q \rightarrow T_{g}$ be the bundle of holomorphic quadratic differentials over Teichmüller space, $\mathcal{C}$ the universal curve which fibers over Teichmüller space as $R: \mathcal{C} \rightarrow T_{g}$, and $S: R^{*} Q \rightarrow \mathcal{C}$ the pull back bundle of holomorphic quadratic differentials over $\mathcal{C}$. Our discussion of the previous paragraph provides for a canonical family in the base $\mathcal{C}$ corresponding to a neighborhood in $T_{g}$. Moreover, we have a map $I: R^{*} Q \rightarrow \mathbf{R}_{+}^{6 g-6}$ given by $q \mapsto\left\langle\operatorname{Im} \int_{\gamma_{i}} \sqrt{q}\right\rangle$ : here, the transversality of the curves $\gamma_{i}$ permits us to make consistent choices of the square root. It is easy to check that conditions (i) and (ii) provide that this map is differentiable; the differential $\left.D I(\Phi)\right|_{S^{-1}(M, \sigma)}$ at $\Phi$ has a restriction to the fiber of full rank because of condition (iii). Thus, we may apply the implicit function theorem to obtain a differentiable section $E_{(\mathcal{F}, \mu)}$ of $S: R^{*} Q \rightarrow \mathcal{C}$ which has horizontal measured foliations equivalent to $(\mathcal{F}, \mu)$. This differentiability of $E_{(\mathcal{F}, \mu)}$ provides for the zeros of the section to vary differentiably, which of course implies that $\dot{a}(\zeta)$ is bounded, as required. 
We continue to assume that $(\mathcal{F}, \mu)$ has only simple zeros unconnected by leaves of $\mathcal{F}$, and prove

Lemma 4.12. For $t$ sufficiently small, $E^{*}\left(\rho_{t}\right)>E^{*}\left(\rho_{0}\right)$ for $t \neq 0$.

Proof. From the previous proof, we observe two facts: (i) $F_{\rho_{t}}$ is $C^{\infty}$ off of a neighborhood of the zeros of $\Phi_{\rho_{t}}$ (ii) the infinitesimal normal variation is $O\left(|\zeta|^{1 / 2}\right)$ for $\zeta$ a conformal coordinate centered at a zero of $\Phi_{\rho_{0}}$.

We consider the surface with boundary $M_{\delta}$ defined as the complement in $M$ of $\delta$-neighborhoods of the zeros of $\Phi_{\rho_{0}}$, measured with respect to, say, the metric $\rho_{0}$. We apply the formulas (4.5), (4.9) to the Hessian for (equivariant) area on $\widetilde{M}_{\delta}$ : from formula (4.9), we see that the result is a term which is positive (compare (ii) and (4.11)) and a boundary term coming from applying Stokes' theorem to the divergence term. Yet this boundary term is a sum of terms of the form

$$
\int_{\partial B_{\delta}}\left[O\left(|\zeta|^{1 / 2}\right)\right]^{2} \frac{\partial}{\partial n} \log \frac{1-|\tau|}{1+|\tau|}
$$

which vanishes as $\delta \rightarrow 0$ (here $|\tau|=O(|\zeta|)$ ).

Finally, we observe that this induced normal field on $\widetilde{M}_{\delta}$ does not vanish identically, for if it $\operatorname{did}$, the map $\widetilde{w}_{t}:\left(\widetilde{M}, \widetilde{\rho}_{t}\right) \rightarrow(\widehat{N}, \widetilde{h})$ would induce an equivariant map $\widetilde{G}_{t}: F_{t}(\widehat{M}) \rightarrow F_{0}(\widetilde{M})$ which would then be infinitesimally conformal. But the image $F_{0}(\widetilde{M})$ is equivariantly conformal to $(\widetilde{M}, \tilde{\sigma})$, and, by the construction of $\left(\widetilde{M}, \widetilde{\rho}_{t}\right)$ in Teichmüller space, is then conformally distinct, even infinitesimally, from $\left(\widetilde{M}, \widetilde{\rho}_{t}\right)$. However, if we assume in addition the contrary of the statement of the lemma, i.e., that $E^{*}\left(\rho_{t}\right)=E^{*}\left(\rho_{0}\right)$ (using Lemma 4.9 to exclude $\left.E^{*}\left(\left[\rho_{t}\right]\right)<E^{*}\left(\left[\rho_{0}\right]\right)\right)$, then the path $\left\{\left[\rho_{t}\right]\right\}$ would be a path of critical points for $E^{*}$, forcing $\left(\widetilde{M}, \widetilde{\rho}_{t}\right)$ to be equivariantly conformal to $F_{t}(\widetilde{M})$, by Lemma 4.9 . Thus, $\left(M, \rho_{t}\right)$ would be infinitesimally conformal to $(M, \sigma)$, a contradiction.

Thus, applying (i) so that (4.5) represents the Hessian of area of $F_{\rho_{t}}\left(M_{\delta}\right)$, we see that the area $A\left(F_{\rho_{t}}(M)\right)$ satisfies

$$
A\left(F_{\rho_{t}}(\widetilde{M})\right)>A\left(F_{\rho_{0}}(\widetilde{M})\right)+c t^{2}
$$

for some constant $c>0$.

Of course, by the arithmetic-geometric inequality, since $E^{*}\left(\rho_{t}\right)$ represents the (equivariant) energy of $F_{\rho_{t}}$, we see that $E^{*}\left(\rho_{t}\right) \geq A\left(F_{\rho_{t}}(\widetilde{M})\right)$ 
so that $E^{*}\left(\rho_{t}\right)>A\left(F_{\rho_{0}}(\widetilde{M})\right)+c t^{2}=E^{*}\left(\rho_{0}\right)+c t^{2}$; the last equality follows from the conformality of $F_{\rho_{0}}$. q.e.d.

Finally, we prove our uniqueness statement:

Proposition 4.13. For every measured foliation $(\mathcal{F}, \mu)$ with only simple zeros unconnected by leaves of $\mathcal{F}$, there is a unique conformal structure $(M, \sigma)$ so that the Hopf differential for the harmonic map $f_{\sigma}$ : $(M, \sigma) \rightarrow(N, h)$ has maximal stretch foliation $(\mathcal{F}, \mu)$.

Proof. Existence was proved in $\S 3$. The present issue is uniqueness, and the idea is to apply a qualitative Morse theory on $T_{g}$ to the function $E^{*}: T_{g} \rightarrow \mathbf{R}$.

By Proposition 4.9, any such conformal structure $(M, \sigma)$ is a critical point of $E^{*}$. Suppose that there are two such distinct structures $(M, \sigma)$ and $\left(M, \sigma^{\prime}\right)$. Now $E^{*}$ is a proper $C^{1}$ function on $T_{g}$, and so a variant (discussed next) of the classical "mountain pass lemma" will then assert the existence of a critical point $\left(M, \sigma^{\prime \prime}\right)$ for $E^{*}$ which is not stable for $E^{*}$. This then contradicts Lemma 4.12 , proving the proposition.

A sufficiently general such mountain pass lemma may be found (Theorem II.1.12) in Struwe's book [20], where it is stated for Palais-Smale functions on some (possibly) infinite-dimensional manifolds; it is easy to check that our present situation easily satisfies the hypotheses. While it would be a bit distracting to reproduce the proof here, we recall that we may locate $\left(M, \sigma^{\prime \prime}\right)$ as that point in Teichmüller space which satisfies the minimax characterization

$$
E^{*}\left(\sigma^{\prime \prime}\right)=\inf _{\gamma \in \mathcal{G}} \sup _{p \in \gamma} E^{*}(p)
$$

where $\mathcal{G}=\left\{\gamma \in C^{1}\left([0,1], T_{g}\right): \gamma(0)=\sigma, \gamma(1)=\sigma^{\prime}\right\}$. q.e.d.

4.3.2. Case 2. In our proofs of Lemma 4.10, Lemma 4.12 and Proposition 4.13 , we only used the hypothesis that $\widetilde{\Phi}$ had only simple zeros to show that any deformation $F_{\rho_{t}}$ of $F_{\rho_{0}}$ was strongly admissable, so that the Hessian of area was positive definite at $\rho_{0}=\sigma$. This argument involved a computation of the order of vanishing of the normal $\dot{n}$ at a zero of $\widetilde{\Phi}$, which in turn was based on a computation of the smoothness of the Hubbard-Masur section $E_{(\mathcal{F}, \mu)}$ of $R^{*} Q \rightarrow \mathcal{C}$ corresponding to the measured foliation $(\mathcal{F}, \mu)$, as well as a formal differentiation of a family of transverse measures.

For the case of higher order zeros, the outline of the argument is unchanged, but the technicalities become more complicated. The principal 
difficulty is that the map $I: R^{*} Q \rightarrow \mathbf{R}_{+}^{6 g-6}$ to which we applied the implicit function theorem to in the previous case, may lack a uniform $C^{1}$ bound as a sequence $\Phi_{n}$ of holomorphic quadratic differentials with simple zeros approaches a holomorphic quadratic differential $\Phi_{0}$ with higher order zeros.

To see that this is really our principal difficulty, it is instructive (and relevant) to proceed with the proof, assuming for now the differentiability of the section $E_{(\mathcal{F}, \mu)}$, where $(\mathcal{F}, \mu)$ represents a foliation corresponding to a holomorphic quadratic differential $\Phi_{0}$ with higher order zeros.

Away from the zeros of $\Phi_{0}$, we can consider our map $p_{\rho_{t}}:\left(\widetilde{M}, \widetilde{\rho_{t}}\right) \rightarrow$ $(T, 2 d)$ as locally a differentiable family of harmonic functions. Thus $d\left(p_{\rho_{t}}(A), p_{\rho_{t}} \widetilde{\Phi}_{0}^{-1}(0)\right)$ is differentiable in $t$, for a fixed point $A$. We compute (in our normalized coordinates), still under our assumption of the differentiability of $E_{(\mathcal{F}, \mu)}$ and in analogy to (4.14),

$$
\begin{aligned}
& \left\|\pi_{2 *}\left[\left.\frac{d}{d t}\right|_{t=0} F_{\rho_{t}}(\zeta)\right]\right\| \\
& \quad=\left|\frac{d}{d t}\right|_{t=0} \int_{a(t)}^{\zeta} \operatorname{Im} \sqrt{\Psi_{t}} \mid \\
& \quad=\left|\frac{d}{d t}\right|_{t=0} \int_{a(t)}^{\zeta} \operatorname{Im} \sqrt{z^{k}+P_{k-2}(z, t)} d z \mid,
\end{aligned}
$$

where $P_{k-2}(z, t)$ is a polynomial of order $k-2$ in $z$. Here we can assume that $\Psi_{t}=z^{k}+t P_{k-2}(z)$ because by $([12$, Theorem 3.2 , see also Proposition 3.1]), the space of degree $k-2$ polynomials contains the possible infinitesimal deformations of the local form $\Psi_{0}$, up to pullback by a diffeomorphism

Thus if the coefficients of $P_{k-2}(z, t)$ are differentiable in $t$, as we are assuming for now that they are, the right-hand side is either $O(\log |(\zeta)|)$ or $O\left(|\zeta|^{-\frac{k}{2}+1}\right)$ depending on whether $k=2$ or $k \neq 2$, respectively. But then, as this quantity (4.15) is comparable to $|\dot{n}(\zeta)|$, we see that $|\dot{n}(\zeta)||\zeta|^{\frac{k}{2}}=o(1)$, as is required for an admissable deformation. By Theorem 4.3 , then the minimal suspension is stable; finally, it is easy to check that the exceptional case in Corollary 4.7 cannot occur for $k \geq 2$, so that the second variation of area is even positive.

Hence we are led to study the differentiablity of the section $E_{(\mathcal{F}, \mu)}$ over $T_{g}$ more carefully. This study is the bulk of the work of Hubbard and Masur ([12]) in their argument for the existence of a well-defined 
section $E_{(\mathcal{F}, \mu)}$, and we will be able to retrieve the estimates we need from [12].

We begin by observing that if we use a path in $T_{g}$ to induce a deformation $F_{t}:(-\eta, \eta) \times \widetilde{\mathcal{R}} \rightarrow \widetilde{N} \times T$, we are not required to use, in our computation of $\dot{n}$, the differentiable structure of that path that is induced by its embedding in $T_{g}$ : it could well be that an exotic differentiable structure could produce an infinitesimal deformation $\dot{n}$ that is more tractable for us than that induced by the standard differentiable structure (See [12, Theorem 3.2]). Put differently, a curve in $T_{g}$ induces a family of surfaces in $\widetilde{N} \times T$ which we are free to parametrize to our convenience. With that in mind, we recall that a principal technical tool of [12] is their construction of exotic (but convenient) differentiable structures for spaces of deformations of holomorphic quadratic differentials with high multiplicities. (We will need weaker results than those in [12], as we will require only some diffentiability along a curve in $T_{g}$ instead of the existence of a (close to continuous) differential, and we already know the existence of the section $E_{(\mathcal{F}, \mu)}$.) Indeed, in terms of these structures ([12, Step 2 (p. 261); Proposition 4.11, Lemma 4.12 (p. 269) and the references therein; as well as the example on p. 241 for motivation]) on curves in $T_{g}$, the section $E_{(\mathcal{F}, \mu)}$ will be differentiable, and so our computations in the first full paragraph after (4.15) will be valid.

Here is one way to produce this exotic differentiable structure, along the lines of [12, see p. 241]. Again, for the sake of clarity, we first treat a particular class of curves, and then generalize to all the curves we need. Along a curve $B \subset T_{g}\left(B=\left\{\rho_{t}\right\}\right.$ to be chosen more carefully in a moment) through $\left[\rho_{0}\right]$ lying under $\left(\left[\rho_{0}\right], \Phi_{0}\right)$, embed $Q$ in $Q \times \mathbf{R}^{N}$ by $J: q \mapsto\left(q,\left\langle\int_{\gamma_{i}} \operatorname{Im} \sqrt{q}\right\rangle_{i=1}^{6 g-6}\right)$; here as in the first case, $\left\langle\gamma_{1}, \ldots, \gamma_{6 g-6}\right\rangle$ are curves on $M$ which (i) are transverse to the horizontal foliation of $E_{(\mathcal{F}, \mu)}\left(\left[\rho_{t}\right]\right)$ near the zero set of $E_{(\mathcal{F}, \mu)}\left(\left[\rho_{t}\right]\right)$ (for $\left[\rho_{t}\right]$ distinct from $\left[\rho_{0}\right]$ ), and (ii) have measures which provide local coordinates for $\mathcal{M F}$ near $(\mathcal{F}, \mu)$. We give $E_{(\mathcal{F}, \mu)}$ the differentiable structure induced by its embedding as a graph by $J$. In particular, away from $\left[\rho_{0}\right]$ along $\mathrm{B}$ in the (generic) set where $E_{(\mathcal{F}, \mu)}$ has simple zeros, the map $I: q \mapsto\left\langle\operatorname{Im} \int_{\gamma_{i}} \sqrt{q}\right\rangle$ is differentiable at $E_{(\mathcal{F}, \mu)}\left(\left[\rho_{t}\right]\right)$, and the effect of our parameterization by $J$ is to force (away from $\left(\left[\rho_{0}\right], \Phi_{0}\right)$ ) uniform bounds on both $\left\|\left.D I\right|_{B}\right\|$ and $\left\|\left.D I(\Phi)\right|_{S^{-1}\left(M, \rho_{0}\right)}\right\|^{-1}$ : the implicit function theorem then guarantees a uniform bound on $D E_{(\mathcal{F}, \mu)}[V]$, away from $\left[\rho_{0}\right]$, where $V$ is 
tangent to the curve $\mathrm{B}$ at $\left[\rho_{t}\right] \in B$. This bound then extends to a bound, in the new differentiable structure, on $\left.D E_{(\mathcal{F}, \mu)}\right|_{\left[\rho_{0}\right]}[V]$ (where $V$ is again tangent to $B$ at $\left[\rho_{0}\right]$, with tangent vector defined by the new structure), as desired. We use this structure to undertake the computations of (4.15); observe that in this exotic differentiable structure, the construction guarantees that the vector field $\dot{n}$ will not vanish, even if the conformal structure $\left[\rho_{t}\right]$ of the underlying surface does not change infinitesimally.

Now, the section $E_{(\mathcal{F}, \mu)}$ stratifies in terms of the number of zeroes and their orders. In terms of this stratification, the arguments in Case 1 (Section 4.3.2) were for $\left[\rho_{0}\right]$ an interior point of the (single) principal stratum, and in the last paragraph we discussed curves in the principal stratum which met another stratum at $\left[\rho_{0}\right]$. In fact, for curves in $T_{g}$ through $\left[\rho_{0}\right]$ for which the complement of $\left[\rho_{0}\right]$ is contained in a single lower stratum, the identical argument as for the last paragraph holds: we need only choose our curves $\gamma_{i}$ to be transverse to the foliation of $E_{(\mathcal{F}, \mu)}\left(\left[\rho_{t}\right]\right)$, for $\left[\rho_{t}\right] \in B$. (These curves $\gamma_{i}$ then may possibly pass through the zero locus of $E_{(\mathcal{F}, \mu)}\left(\left[\rho_{t}\right]\right)$, but we observe that this does not affect the argument.)

We summarize the situation as follows: the discussion of the previous paragraphs shows that for $B$ a differentiable curve in $T_{g}$ which is contained in a single stratum off of $\left[\rho_{0}\right]$, we find that we can find a coordinate $t$ on $B$ so that $B=\left\{\left[\rho_{t}\right]\right\}$ with

$$
A\left(F_{\rho_{t}}(\widetilde{M})\right)>A\left(F_{\rho_{0}}(\widetilde{M})\right)+c t^{2}
$$

where $c$ depends on the curve $B$ in $T_{g}$; here $c$ varies continuously as the curve varies continuously in the stratum. Now, inequality (4.16) holds for all such curves (with a constant depending on the curve), and every point in a neighborhood of $\left[\rho_{0}\right]$ is contained in one of these curves ([12, $\S \S[V .1$, IV.5]); moreover, these curves have well-defined initial directions corresponding to the infinitesimal strata ([12, $\S \S \mathrm{IV} .1, \mathrm{IV} .5]$, again), and the space of initial directions for these curves is compact, while the space of strata is finite. We conclude that (4.16) holds (for some constant $c$, and $\left[\rho_{t}\right]$ along one of our curves) for a neighborhood in $T_{g}$. This proves the analogue of Lemma 4.12 for an arbitrary foliation, while the analogue of Proposition 4.13 is unchanged. This completes the proof of uniqueness, hence Theorem 3.1. q.e.d. 


\section{References}

[1] S. I. Al'ber, On n-dimensional problems in the calculus of variations, Soviet Math. Dokl. 5 (1964) 700-704.

[2] L. Ahlfors, Some remarks on Teichmüller's space of Riemann surfaces, Ann. of Math. 74 (1961) 171-191.

[3] J. Eells \& L. Lemaire, Deformations of metrics and associated harmonic maps, Patodi Mem. Vol. Geom. Anal. (Tata Inst.) 1981, 33-45.

[4] J. Eells \& J.H. Sampson, Harmonic mappings of Riemannian manifolds, Amer. J. Math. 86 (1964) 109-160.

[5] A. Fathi, F. Laudenbach \& V. Poenaru, Traveaux de Thurston sur les Surfaces, Asterisque (1979) 66-67.

[6] A. Friedman, Partial differential equations, Holt, Rinehart and Winston, New York, 1969.

[7] F. P. Gardiner, Measured foliations and the minimal norm property for quadratic differentials, Acta Math. 152 (1984) 57-76.

[8] 1987.

[9] F. P. Gardiner \& H. Masur, Extremal length geometry of Teichmüller space, Complex Anal. Appl. 16 (1991) 209-237.

[10] M. Gromov \& R. Schoen, Harmonic maps into singular spaces and p-adic superrigidity for lattices in groups of rank one, Inst. Hautes Études Sci. Publ. Math. 76 (1992) 165-246.

[11] P. Hartman, On homotopic harmonic maps, Canad. J. Math. 19 (1967) 673-687.

[12] J. Hubbard \& H. Masur, Quadratic differentials and foliations, Acta Math. 142 (1979) 221-274.

[13] J. Jost, Two dimensional geometric variational problems, Wiley Intersci., New York, 1991.

[14] S. Kerckhoff, The asymptotic geometry of Teichmüller space, Topology 19 (1980) 23-41.

[15] Y. Minsky, Harmonic maps, length, and energy in Teichmüller space, J. Differential Geom. 35 (1992) 151-217.

[16] - Teichmüller geodesics and ends of hyperbolic 3-manifolds, Topology 32 (1993) 625-647.

[17] D. Mumford, A remark on Mahler's compactness theorem, Proc. Amer. Math Soc. 28 (1971) 288-294. 
[18] J. H. Sampson, Some properties and applications of harmonic mappings, Ann. Sci. École Norm. Sup. 4 (1978) 265-278.

[19] R. Schoen, Stanford Lecture Notes, 1983.

[20] M. Struwe, Plateau's problem and the calculus of variations, Princeton Univ. Press, Princeton,1989, Princeton Math. Notes, No. 35.

[21] R. Schoen \& S.-T. Yau, On univalent harmonic maps between surfaces, Invent. Math. 44 (1978) 265-278.

[22] _ Existence of incompressible minimal surfaces and the topology of three dimensional manifolds with non-negative scalar curvature, Ann. of Math. 110 (1979) 127-142.

[23] B. Tabak, A geometric characterization of harmonic maps between surfaces, Math. Ann. 270 (1985) 147-157.

[24] A. Tromba, A new proof that Teichmüller space is a cell, Trans. Amer. Math Soc. 303 (1990) 257-262.

[25] — Teichmüller theory in Riemannian geometry, Birkhäuser, Berlin, 1992.

[26] M. Wolf, The Teichmüller theory of harmonic maps, J. Differential Geom. 29 (1989) 449-479.

[27] - High energy degeneration of harmonic maps between surfaces and rays in Teichmüller space, Topology 30 (1991) 517-540.

[28] — Harmonic maps from surfaces to R-trees, Math. Z. 218 (1995) 577-593.

[29] - On realizing measured foliations via quadratic differentials of harmonic maps to R-trees, J. Analyse Math 68 (1996) 107-120.

[30] S. Wolpert, Geodesic length functions and the nielsen problem, J. Differential Geom. 25 (1987) 275-295.

Added in Proof:

[31] W. Thurston, The geometry and topology of 3-manifolds, Princeton University Lecture Notes, 1979.

[32] F. Bonahon, Bouts des variétés hyperboliques de dimension 3, Ann. of Math. 124 (1986) $71-158$.

Rice University, Houston 